\title{
Early stages of clathrin aggregation at a membrane in coarse-grained simulations
}

M. Giani, W. K. den Otter, and W. J. Briels

Citation: The Journal of Chemical Physics 146, 155102 (2017); doi: 10.1063/1.4979985

View online: http://dx.doi.org/10.1063/1.4979985

View Table of Contents: http://aip.scitation.org/toc/jcp/146/15

Published by the American Institute of Physics

\section{Articles you may be interested in}

Perspective: Dissipative particle dynamics

The Journal of Chemical Physics 146, 150901 (2017); 10.1063/1.4979514

Potential of mean force for insertion of antimicrobial peptide melittin into a pore in mixed DOPC/DOPG lipid bilayer by molecular dynamics simulation

The Journal of Chemical Physics 146, 155101 (2017); 10.1063/1.4979613

Phase behavior of supported lipid bilayers: A systematic study by coarse-grained molecular dynamics simulations

The Journal of Chemical Physics 146, 154902 (2017); 10.1063/1.4981008

The attachment of $\alpha$-synuclein to a fiber: A coarse-grain approach

The Journal of Chemical Physics 146, 115102 (2017); 10.1063/1.4978297

Probing the effects of surface hydrophobicity and tether orientation on antibody-antigen binding

The Journal of Chemical Physics 146, 155103 (2017); 10.1063/1.4980083

Electrolyte solutions at curved electrodes. I. Mesoscopic approach

The Journal of Chemical Physics 146, 154703 (2017); 10.1063/1.4979947

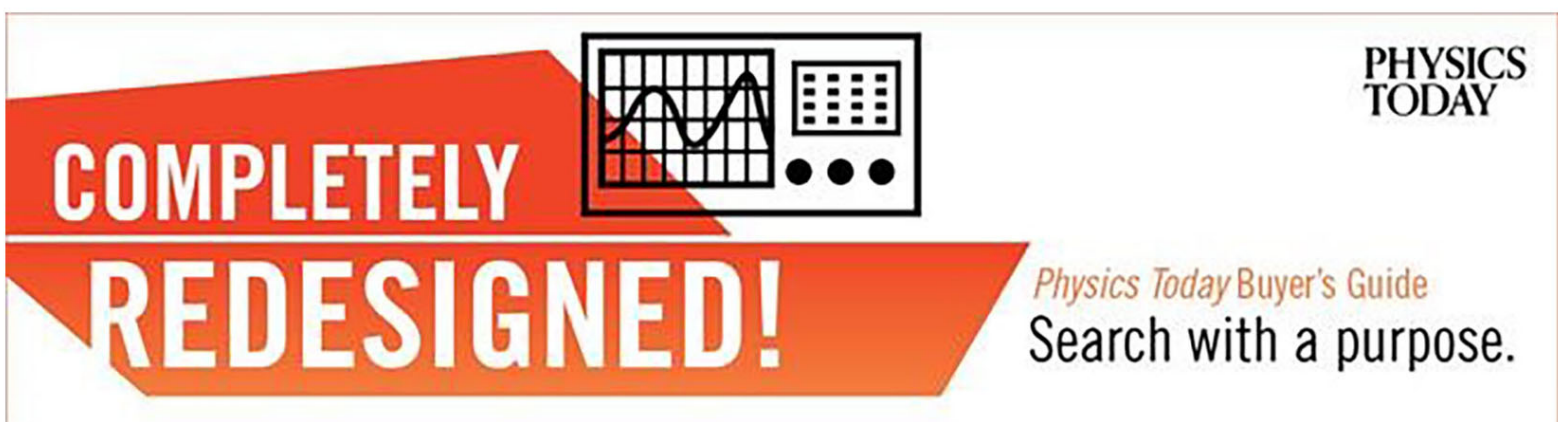




\title{
Early stages of clathrin aggregation at a membrane in coarse-grained simulations
}

\author{
M. Giani, ${ }^{1,2,3}$ W. K. den Otter, ${ }^{1,2,3, a)}$ and W. J. Briels ${ }^{2,3,4}$ \\ ${ }^{1}$ Multi Scale Mechanics, Faculty of Engineering Technology, University of Twente, P.O. Box 217, \\ 7500 AE Enschede, The Netherlands \\ ${ }^{2}$ Computational BioPhysics, Faculty of Science and Technology, University of Twente, P.O. Box 217, \\ 7500 AE Enschede, The Netherlands \\ ${ }^{3}$ MESA+ Institute for Nanotechnology, University of Twente, P.O. Box 217, 7500 AE Enschede, The Netherlands \\ ${ }^{4}$ Forschungszentrum Jülich, ICS 3, D-52425 Jülich, Germany
}

(Received 12 December 2016; accepted 28 March 2017; published online 20 April 2017)

\begin{abstract}
The self-assembly process of clathrin coated pits during endocytosis has been simulated by combining and extending coarse grained models of the clathrin triskelion, the adaptor protein AP2, and a flexible network membrane. The AP2's core, upon binding to membrane and cargo, releases a motif that can bind clathrin. In conditions where the core-membrane-cargo binding is weak, the binding of this motif to clathrin can result in a stable complex. We characterize the conditions and mechanisms resulting in the formation of clathrin lattices that curve the membrane, i.e., clathrin coated pits. The mechanical properties of the AP2 $\beta$ linker appear crucial to the orientation of the curved clathrin lattice relative to the membrane, with wild-type short linkers giving rise to the inward curving buds enabling endocytosis while long linkers produce upside-down cages and outward curving bulges. Published by AIP Publishing. [http://dx.doi.org/10.1063/1.4979985]
\end{abstract}

\section{INTRODUCTION}

Eukaryotic cells possess the remarkable ability to collect, sort, and internalize a variety of membrane components and external cargo molecules, by a process known as clathrin-mediated endocytosis (CME) ${ }^{1-3}$ The two main proteins involved in CME are clathrin and the AP2 adaptor protein complex, assisted by a series of accessory proteins. ${ }^{4}$ Clathrin is a three-legged protein, see Fig. 1, with the ability to self-assemble into a variety of polyhedral cages in vivo and in vitro. ${ }^{5,6}$ In cages, a clathrin triskelion is centered at every vertex, with each of its legs running along two edges before bending inward at the leg's terminal domain (TD). ${ }^{7-9}$ Cages grown in vivo enclose lipid vesicles, with the clathrin triskelia tethered to the membrane by multiple AP2 complexes. The latter carries motifs for the phosphatidylinositol 4,5-bisphosphate $\left[\operatorname{PtdIns}(4,5) \mathrm{P}_{2}\right]$ lipids specific to the plasma membrane ${ }^{10}$ and motifs binding cargo molecules, all located in the folded core of the protein, as well as two binding sites for clathrin on a long flexible linker. ${ }^{1-13}$ Crystallography experiments indicate that the mid-linker motif binding a clathrin $\mathrm{TD}^{9}$ is only released by the AP2 core when that core has bound $\operatorname{PtdIns}(4,5) \mathrm{P}_{2}$ and cargo, ${ }^{14,15}$ while earlier binding essays suggest that the permanently available "ear" or "appendage" site at the free end of the linker binds a site higher up the clathrin leg. ${ }^{16}$ In the highly coordinated process of endocytosis, ${ }^{17}$ AP2 intermediates by bringing cargo and triskelia together in a clathrin coated pit (CCP), i.e., a membrane invagination coated by a clathrin lattice. As the lattice continues to grow and curve, the membrane is wrapped until the above mentioned clathrin

a)w.k.denotter@utwente.nl coated vesicle $(\mathrm{CCV})$ is pinched off from the membrane. Finally, the proteins decorating the vesicle are released for the next cycle while the vesicle carries the cargo to an organelle for further processing. Clathrin also produces transport vesicles at other sites in the cell, in collaboration with a wide range of membrane-specific and/or cargo-specific adaptor proteins. ${ }^{11,18,19}$

Current experimental techniques do not permit examination at the molecular level of CCP formation in living cells. Cryo-electron microscopy produces high resolution stills of CCPs at various stages of maturation, thereby providing insights into the structure of these $\mathrm{CCPs}^{20}$ as well as revealing the presence of large flat clathrin lattices. ${ }^{21,22}$ Labeling selected proteins with fluorescent labels enables imaging endocytosis in living cells. ${ }^{23}$ Fluorescence recovery after photobleaching (FRAP) reveals that triskelia in pits are readily exchanged, unlike those in cages grown in vitro. ${ }^{24-26}$ Total internal reflection fluorescence (TIRF) microscopy unravels the sequence of arrival and departure of various proteinsincluding cargo, clathrin, adaptor proteins, and the membrane scission protein dynamin - over the course of approximately 1 min between the initiation and successful conclusion of an endocytic event. ${ }^{27-30}$ Besides initiations that mature to completion, these experiments also detect aborted events. ${ }^{31,32}$ In two recent studies, single particle tracking is combined with photo-activation localization microscopy (PALM) to follow the internalization by CME of nano-particles in unparalleled resolution ${ }^{33}$ and fluorescence intensity tracing is used to shift the focus from the plasma membrane-coverglass interface to CCP formation at unrestrained membranes. ${ }^{34}$ Despite the wealth of information obtained by these and other optical techniques, a number of fundamental questions on CCP dynamics 


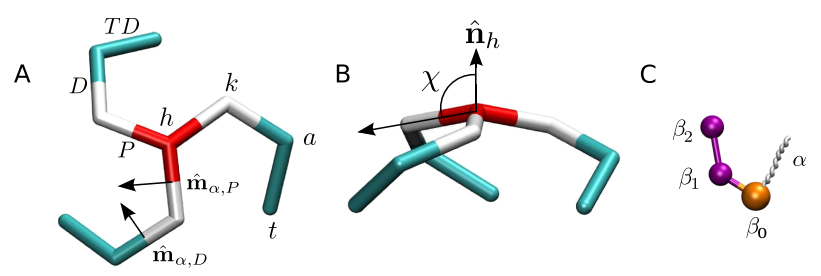

FIG. 1. The highly coarse-grained models used for simulating clathrin ((A) and (B)) and AP2 (C), plotted on the same scale. In the rigid clathrin triskelion, three proximal leg segments $(\mathrm{P})$ radiate from a central hip $(\mathrm{h})$ to the knees $(\mathrm{k})$, at a pucker angle $\chi$ relative to the symmetry axis $\hat{\mathbf{n}}_{h}$, followed by distal leg segments (D) running to ankles (a) and terminal domains (TDs) ending at the toes $(\mathrm{t})$. The directionality of leg-leg interactions, represented by the polarity vectors $\hat{\mathbf{m}}$, is elaborated in Fig. 2. The AP model features three beads connected by two flexible linkers: the $\beta_{1}$ and $\beta_{2}$ beads can bind to the toes and ankles of clathrin, respectively, while the core $\beta_{0}$ can bind to a membrane bead. The full AP2 protein also possesses a flexible $\alpha$ linker, which is omitted in the simulations as it does not bind to membranes nor to clathrin.

are still hotly debated. These include the mechanical contribution of clathrin toward bending the membrane, ${ }^{35-38}$ and whether CCPs are formed by nucleation and growth or by gradually increasing the curvature of a pre-existing planar lattice. . $^{20,26,33,34,39-43}$

Computer simulations provide a powerful tool to explore the statistical-mechanical feasibility of hypothesized selfassembly pathways of CCPs, based on experimentally deduced and/or estimated interactions between the key proteins. Matthews and Likos modelled clathrin as a triskelion composed of 13 bead particles with interaction patches on their surfaces, showing that these triskelia can self-assemble into cages as well as create deep membrane invaginations. ${ }^{44}$ Spakowitz and collaborators modelled clathrin as a particle that forms harmonic bonds with three neighbours to study the mechanical properties of lattices against a flat membrane and the fluidization of these lattices when the membrane is nanoindented. ${ }^{45,46}$ Adaptor proteins were omitted in both types of simulations, by enabling the clathrin particles to bind directly to the membrane. The indirect coupling of triskelia to the membrane in cells - note that the disordered linkers of adaptor proteins transmit pulling forces but not pushing forces-is likely to strongly affect the deformation of the membrane by the coat. We have developed a simulation model of clathrin as a rigid particle with kinked legs, see Fig. 1, and with this model showed that asymmetric leg-leg interactions hold the key to self-assembly, related the binding energy to the critical assembly concentration (CAC), established a time scale for self-assembly in solution, and observed how flat lattices release early CCPs when forced to curve. ${ }^{47-50}$ In a recent study, we developed a simulation model for AP2. ${ }^{51}$ Several combinations of AP-binding locations on the clathrin leg were explored to separate combinations that enable cage assembly from those that do not, thereby arriving at the insight that the entropic spring linking the two clathrin motifs of AP2 is crucial to the functioning of this adaptor protein. The combination of both binding sites residing at the TD, which was not explicitly mentioned in that paper, did not prove able to promote cage assembly in the bulk. This may explain the recent observation by Moshkanbaryans et al. ${ }^{52}$ that a newly discovered "site 1" on AP180 binds weaker to clathrin than eight previously identified TD-binding motifs yet proves crucial to
AP-induced cage formation, by the hypothesis that site 1 is the only motif that binds clathrin at a location higher up the leg.

Modeling any process, especially one as complex as CME, involves making assumptions-based on the available experimental data-regarding the features to be included in the model and the details that can be ignored. In the current study, we explore the early stages of CCP formation for the simplest experimentally functional combination of components: clathrin, membrane, and an adaptor protein. ${ }^{15,37}$ The clathrin protein is modelled as a rigid triskelion with smooth leg-leg interactions. Based on $10^{6}$ triskelia in a cellular volume of $10^{3} \mu \mathrm{m}^{3}$, the clathrin concentration is estimated at $10^{-6} \mathrm{M} .{ }^{53-55}$ A comparable concentration is used in the simulations, while the critical assembly concentration (CAC) of the model triskelion is about an order of magnitude higher ${ }^{49,51}$ The model for $\mathrm{AP} 2$ is equipped with two clathrin binding sites to enable the protein to bring two triskelia together and thereby induce cage assembly, as observed in in vitro experiments..$^{5}$ Our simulations of this process indicated that adaptor proteins - and, by implication, dimers of adaptor proteins ${ }^{56-58}$-are ineffectual when binding to terminal domains only because of the relatively large distance between TDs in a cage. Hence we included binding of the $\beta$-linker appendix to the ankle of a triskelion. Because of the modest experimental evidence supporting this second site, ${ }^{15,16,59}$ we also present simulations without this site.

Spontaneous AP2-induced assembly of cages in solution is prevented by incorporating the switching mechanism proposed by Owen and co-workers, ${ }^{14,15}$ whereby a clathrin binding site on the $\beta$-linker is released only when the AP2's core is bound to membrane, PtdIns $(4,5) \mathrm{P}_{2}$, and cargo. Alternative mechanisms exist, as, for instance, in neurons where the influx of $\mathrm{Ca}^{2+}$ ions triggers the temporary unlocking by dephosphorylation of a collection of adaptor and accessory proteins crucial to CME, collectively known as dephosphins. ${ }^{60}$ Recent studies indicate that FCHo and Eps15 form a complex with AP2 at the membrane, at least in the initial stages of CME, and thereby induce AP2's conformational change that releases the clathrin motif on the $\beta$ linker. ${ }^{61,62}$ The common feature of these mechanisms, i.e., AP2's ability to bind clathrin changes when AP2 binds to the membrane and cargo, is incorporated in our simulation model. For reasons of simplicity, we do not include auxiliary proteins vying with AP2 for the binding sites on the triskelia. 9,63 Nor do we include proteins that promote membrane bending, like BAR-domains and CALM, ${ }^{64-66}$ or proteins that resist membrane bending, like crowding effects by bulky cargo proteins. ${ }^{38,67}$ The model we have build, guided by the experimental data on a diverse set of proteins with complex interactions, is a highly coarse-grained idealization involving only clathrin, AP2, and membrane beads, to gain a deeper understanding by statistical mechanical simulations of the minimum requirements for the formation of clathrin coated pits.

The simulation models for clathrin, AP2, and a lipid membrane are introduced in Section II. In Section III, the simulation results are presented. The main conclusions and their biophysical implications are discussed in Section IV. 


\section{MODEL}

In this section, the simulation models for clathrin, AP2, and the membrane are described successively. A discussion of the assumptions made to arrive at the model was presented at the end of Sec. I. The model parameters for the force field and the Brownian Dynamics (BD) propagator are summarized in Tables I and II, the rates of the Monte Carlo (MC) steps are collected in Table III. Readers less interested in the fine details of the model are advised to proceed to the presentation of the results in Section III, where the features of the models will be briefly reiterated, and the discussion of the biological implications in Section IV.

\section{A. Clathrin}

\section{The clathrin model}

In several preceding studies, we modelled clathrin as curved, rigid patchy particles to study the in vitro self-assembly of clathrin cages in bulk through Monte Carlo and Brownian dynamics simulations. ${ }^{47-51}$ We here provide a brief description of the model and refer the reader to our previous publications for a more detailed discussion. The model triskelion consists of three identical legs stemming from a central "hub" (h) at a "pucker" angle $\chi$ relative to the normal vector $\hat{\mathbf{n}}_{h}$ along the threefold rotational symmetry axis of the particle, see Fig. 1. Each leg consists of three segments: the proximal $(\mathrm{P})$ and distal (D) sections and the terminal domain (TD), connected by the knee (k) and ankle (a), respectively. All three leg segments are straight and of identical length, $\sigma=17 \mathrm{~nm}$. The orientation of the distal segments relative to the proximal segments is chosen to allow the maximum overlap between the legs of a triskelion and those of a secondary triskelion whose hub is situated at a knee of the primary triskelion. According to the structural information file 1XI4, ${ }^{8,68}$ available at the Protein Data Bank (PDB), the terminal domain forms an angle of $\sim 114^{\circ}$ with the adjacent distal segment and a dihedral angle of $\sim 28^{\circ}$ relative to the distal and proximal segments of the same leg. We select a pucker angle of $\chi=101^{\circ}$, as this value corresponds to soccer-ball cages containing 60 triskelia, the most commonly

TABLE I. Interaction parameters of the six distinct clathrin leg segment pairings. In the first column, the letters refer to the hub $(h)$, knee $(k)$, and ankle $(a)$ of legs $\alpha$ and $\beta$ of particles $i$ and $j$, respectively. Note that the order is important: the two proximal-proximal pairings, i.e., the first attractive combination and the first repulsive combination, refer to Eqs. (1) and (2), respectively. The elements in the last column represent the arguments $x$ to the polarity function $g(x)$, where the first polarity vector in the dot products refers to a segment of the $\alpha$ leg of particle $i$ and the second polarity vector to a segment of the $\beta$ leg of particle $j$, and where $g(-1)=1$.

\begin{tabular}{lcccc}
\hline \hline$i \boldsymbol{\alpha}-j \beta$ & $\boldsymbol{\varepsilon}$ & $A / \sigma^{-1}$ & $r_{\text {cut }} / \sigma$ & $x$ \\
\hline Attractive & & & & \\
$h k-k h$ & $\boldsymbol{\epsilon}$ & 4 & 0.4 & $\hat{\mathbf{m}}_{P} \cdot \hat{\mathbf{m}}_{P}$ \\
$k a-a k$ & $\epsilon$ & 4 & 0.4 & $\hat{\mathbf{m}}_{D} \cdot \hat{\mathbf{m}}_{D}$ \\
$h k-k a$ & $\epsilon / 2$ & 4 & 0.4 & $-\hat{\mathbf{m}}_{P} \cdot \hat{\mathbf{m}}_{D}$ \\
$h k-a k$ & $\epsilon / 2$ & 0.8 & 0.4 & $\hat{\mathbf{m}}_{P} \cdot \hat{\mathbf{m}}_{D}$ \\
Repulsive & & 0.8 & 0.8 & -1 \\
$h k-h k$ & $-10 \boldsymbol{\epsilon}$ & $-10 \boldsymbol{\epsilon}$ & 0.8 & -1 \\
$k a-k a$ & & & & \\
\hline \hline
\end{tabular}

TABLE II. Summary of the applied simulation parameters for clathrin, AP, and membrane particles, as well as their interaction parameters.

\begin{tabular}{|c|c|c|c|}
\hline Particle & Parameter & Symbol & Value \\
\hline \multirow[t]{8}{*}{ Clathrin } & Segmental length & $\sigma$ & $17 \mathrm{~nm}$ \\
\hline & Pucker & $\chi$ & $101^{\circ}$ \\
\hline & Intersegment energy ${ }^{a}$ & $\epsilon$ & $6 k_{B} T^{\mathrm{b}}$ \\
\hline & Transl. diff. coeff. & $D_{1}^{t}, D_{2}^{t}$ & $4.46 \times 10^{4} \sigma^{2} / \mathrm{s}$ \\
\hline & & $D_{3}^{t}$ & $3.70 \times 10^{4} \sigma^{2} / \mathrm{s}$ \\
\hline & Rotat. diff. coeff. & $D_{1}^{r}, D_{2}^{r}$ & $1.64 \times 10^{4} / \mathrm{s}$ \\
\hline & & $D_{3}^{r}$ & $1.02 \times 10^{4} / \mathrm{s}$ \\
\hline & Bulk concentration $^{c}$ & {$[\mathrm{C}]_{r}$} & $10^{-3} \sigma^{-3}$ \\
\hline \multirow[t]{7}{*}{ AP } & Linker length & $L_{01}$ & $0.9 \sigma$ \\
\hline & & $L_{12}$ & $1.5 \sigma$ \\
\hline & Spring constant & $k_{01}$ & $50 k_{B} T / \sigma^{2}$ \\
\hline & & $k_{12}$ & $30 k_{B} T / \sigma^{2}$ \\
\hline & Transl. diff. coeff. & $D_{0}^{t}$ & $2 \times 10^{4} \sigma^{2} / \mathrm{s}$ \\
\hline & & $D_{1}^{t}, D_{2}^{t}$ & $1 \times 10^{5} \sigma^{2} / \mathrm{s}$ \\
\hline & Bulk concentration & {$[\mathrm{A}]_{r}$} & {$\left[10^{-3}-10^{-2}\right] \sigma^{-3}$} \\
\hline \multirow[t]{7}{*}{ Membrane } & Bending rigidity & $k_{\kappa}$ & {$[10-30] k_{B} T$} \\
\hline & Spring constant & $k_{m}$ & $100 k_{B} T$ \\
\hline & Equilibrium length & $r_{m}^{0}$ & $0.5 \sigma$ \\
\hline & Maximum length & $L_{m}$ & $0.8 \sigma$ \\
\hline & Excluded volume & $\sigma_{m}^{\mathrm{ev}}$ & $0.35 \sigma$ \\
\hline & & $\epsilon_{m}^{\mathrm{ev}}$ & $10 k_{B} T$ \\
\hline & Transl. diff. coeff. & $D_{m}^{t}$ & $10^{2} \sigma^{2} / \mathrm{s}$ \\
\hline \multirow[t]{2}{*}{$\mathrm{AP}-\mathrm{CL}$} & Click strength & $\epsilon_{\mathrm{CA}}$ & {$[6-14] k_{B} T$} \\
\hline & Click radius & $\rho_{\mathrm{CA}}$ & $0.25 \sigma$ \\
\hline \multirow[t]{2}{*}{$\mathrm{AP}-\mathrm{mb}$} & Click strength & $\epsilon_{\mathrm{A} m}$ & {$[6-14] k_{B} T$} \\
\hline & Click radius & $\rho_{\mathrm{A} m}$ & $0.1 \sigma$ \\
\hline \multirow[t]{3}{*}{$m b-X^{d}$} & Repulsion & $\epsilon_{\mathrm{m}}$ & $10^{3} k_{B} T / \sigma^{4}$ \\
\hline & Vertical range & $x_{F}$ & $0.1 \sigma$ \\
\hline & Horizontal range & $x_{G}$ & $1.0 \sigma$ \\
\hline
\end{tabular}

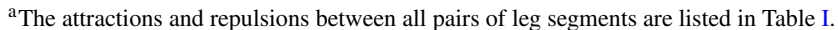

${ }^{\mathrm{b}}$ The experimental data entering the model are based on $T=298 \mathrm{~K}$.

${ }^{\mathrm{c}} 10^{-3} \sigma^{-3} \approx 3.4 \times 10^{-7} \mathrm{M}$.

${ }^{\mathrm{d}} \mathrm{X}=$ clathrin or AP.

observed cage size in in vitro experiments in the presence of AP. ${ }^{5}$

In a completed clathrin cage, a hub is located at every vertex, on top of three knees and three ankles of neighbouring and next-nearest triskelia, respectively, see Fig. 2 . A lattice edge is thus composed of two proximal and two

TABLE III. Attempt rates of all Monte Carlo moves discussed in the main text. The five values in the second half of the table denote rates per particle to eliminate the system size dependence.

\begin{tabular}{lcc}
\hline \hline Function & Symbol & Value $\left(\mathrm{s}^{-1}\right)$ \\
\hline Chemostats, CL, and AP & $r_{c}$ & $10^{5}$ \\
Barostat & $r_{b}$ & $10^{6}$ \\
\hline Membrane bond flipping & $r_{f}$ & $10^{4}$ \\
AP-clathrin click & $r_{\mathrm{CA}}$ & $10^{6}$ \\
AP-membrane click & $r_{\mathrm{A} m}$ & $10^{6}$ \\
Reshuffle clicked AP & $r_{v}$ & $10^{4}$ \\
AP-membrane jumps & $r_{j}$ & $10^{4}$ \\
\hline \hline
\end{tabular}


A

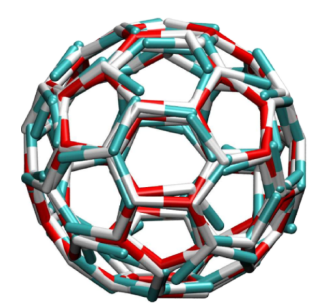

B

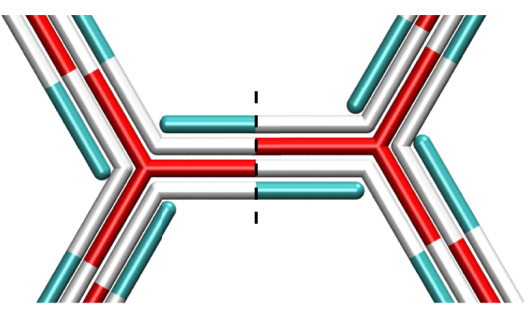

C

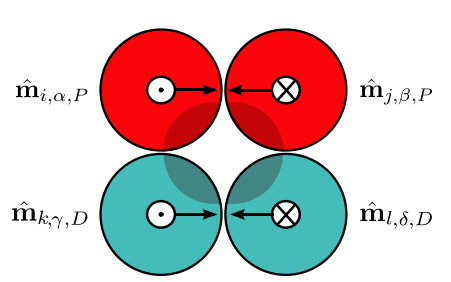

FIG. 2. Cartoons of the cage structure, using the same colouring scheme as in Fig. 1. The terminal domains, curving inward from the ankle toward the center of the cage, have been omitted for clarity. (a) Snapshot of a self-assembled cage in solution. (b) Edges are composed of two proximal and two distal leg segments, with like segments oriented anti-parallel; vertices are meeting points of one hub (red), three knees (white), and three ankles (blue). For clarity, the leg segments are drawn next to each other. (c) The cross section of an experimental cage edge, along the dashed line in (b), with the two distal segments below (i.e., inside the cage) the two proximal segments. The markers $\odot$ and $\otimes$ denote segments pointing (from hip to knee or from knee to ankle) in to and out of the plane of the picture, respectively. Shaded areas highlight the hypothesized locations of binding sites; their asymmetric distribution along the leg's circumference is included in the simulation model by means of a torsion potential acting on the polarity vectors $\hat{\mathbf{m}}$.

distal segments, where the amino acid sequences in both pairs of like segments run in opposite directions (i.e., antiparallel). In our model, the interaction between two triskelia is described by a sum of inter-segmental interactions inspired by the segmental pairings observed in experimental cage edges, and thus attractive interactions are introduced between aligned pairs of two anti-parallel proximal segments, of two anti-parallel distal segments, and between any aligned pair of one proximal and one distal segment-the contribution of the TDs is considered negligible. The attractive interaction between any pair of segments is modelled by a foursite potential based on the distances between the end-points and the orientations of the two segments. All these attractive interactions are anisotropic under rotations around the long axes of the leg segments to mimic that the many weak interaction sites are predominantly located at that side of the leg that faces the neighbouring legs along the same cage edge, see Fig. 2(c). Our previous simulations indicate that this asymmetry or "polarity" holds the key to spontaneous self-assembly of cages. ${ }^{47,48}$

As an example, consider the interaction between the proximal segment of the $\alpha$ th leg of particle $i$ and the proximal segment of the $\beta$ th leg of particle $j$. When the two segments are properly aligned, their respective ends are close to each other. The two average distances between the four ends of these segments are

$$
\begin{aligned}
& r_{j \beta, k h}^{i \alpha, h k}=\frac{1}{2}\left|\mathbf{x}_{i, h}-\mathbf{x}_{j \beta, k}\right|+\frac{1}{2}\left|\mathbf{x}_{i \alpha, k}-\mathbf{x}_{j, h}\right|, \\
& r_{j \beta, h k}^{i \alpha, h k}=\frac{1}{2}\left|\mathbf{x}_{i, h}-\mathbf{x}_{j, h}\right|+\frac{1}{2}\left|\mathbf{x}_{i \alpha, k}-\mathbf{x}_{j \beta, k}\right|,
\end{aligned}
$$

with $\mathbf{x}$ denoting the position of the specific joint indicated in the subscript. The distance on the first line is small if the hub of $i$ is close to the $\beta$ th knee of $j$ and the $\alpha$ th knee of $i$ is close to the hub of $j$ (i.e., aligned and anti-parallel), while the distance on the second line is small if the hub of $i$ is close to the hub of $j$ and the $\alpha$ th knee of $i$ is close to the $\beta$ th knee of $j$ (i.e., aligned and parallel). The former combination occurs in clathrin cages, hence an attractive interaction is assigned,

$$
\phi_{j \beta, k h}^{i \alpha, h k}=-\varepsilon_{k h}^{h k} \cdot f\left(r_{j \beta, k h}^{i \alpha, h k}\right) \cdot g\left(\hat{\mathbf{m}}_{i \alpha, P} \cdot \hat{\mathbf{m}}_{j \beta, P}\right),
$$

with the positive parameter $\varepsilon_{k h}^{h k}$ denoting the (absolute) maximum inter-segmental binding energy. The distance dependence smoothly decreases from unity for coinciding end points to zero at the cut-off distance $r_{\text {cut }}$, following

$$
f(r)=\frac{1}{2}\left[1-\frac{\tanh \left[A\left(r-r_{\mathrm{cut}} / 2\right)\right]}{\tanh \left[A r_{\mathrm{cut}} / 2\right]}\right],
$$

where $A$ determines the steepness of the potential. The numerical values of these parameters are provided in Table I. Although this function favours the proper (anti)parallel alignment of leg segments, it leaves both segments free to rotate around their long axes. The cross section of a cage edge in Fig. 2(c), however, suggests that binding sites are located at one side of the segment and that the segments in a cage present this side to their neighbours. To model this, we associate with every proximal segment a polarity vector, defined for the $\alpha$ th leg as

$$
\hat{\mathbf{m}}_{\alpha, P}=\frac{\hat{\mathbf{n}}_{h} \times\left(\mathbf{x}_{\alpha, k}-\mathbf{x}_{h}\right)}{\left|\hat{\mathbf{n}}_{h} \times\left(\mathbf{x}_{\alpha, k}-\mathbf{x}_{h}\right)\right|},
$$

see Figs. 1(A) and 2. Alignment of the polarities of the two leg segments in Eq. (3) is imposed through

$$
g(x)=\left\{\begin{aligned}
-x & \text { for } x<0 \\
0, & \text { for } x \geq 0
\end{aligned}\right.
$$

All other attractive interactions between segment pairs, see the tabulated list of combinations, are constructed along the same lines. Polarity vectors to distal segment $\hat{\mathbf{m}}_{\alpha, D}$ are defined as in Eq. (5), based on the end points of that segment and the normal at the knee, which is obtained by mirroring the normal vector at the hub in a plane perpendicular to the proximal domain, running through the center of that segment, see Figs. 1 and 2.

Excluded volume interactions between triskelia are omitted for computational reasons. Their introduction would require a more complex particle shape and some flexibility of the legs to enable the particles to interweave four legs along each cage edge. Excluded volume interactions are important in preventing a triskelion from binding to a cage in a position and orientation already occupied by another triskelion. This effect is reproduced by a repulsion between aligned parallel segments of the same type. For two proximal segments, the potential takes the form 


$$
\phi_{j \beta, h k}^{i \alpha, h k}=-\varepsilon_{h k}^{h k} \cdot f\left(r_{j \beta, h k}^{i \alpha, h k}\right),
$$

and likewise for two distal segments, with the parameters listed in Table I. ${ }^{48}$

\section{Propagator}

The trajectories of the triskelia are calculated using first order Brownian dynamics equations of motion for translation and rotation. ${ }^{69-71}$ Since a clathrin triskelion is modelled as a rigid particle, the position and orientation of all leg segments are fully described by the coordinates of its hydrodynamic cen$\operatorname{ter}^{72} \mathbf{r}^{(l)}$ in the laboratory $(l)$ coordinate system and a rotation matrix A relative to the laboratory frame. In the laboratory frame, the coordinates of a site $\alpha$ on the $i$ th particle are then given by

$$
\mathbf{x}_{i \alpha}^{(l)}=\mathbf{r}_{i}^{(l)}+\mathbf{A}_{i} \mathbf{x}_{\alpha}^{(b)}
$$

where $\mathbf{x}_{\alpha}^{(b)}$ represents the constant coordinates of the $\alpha$ th site in the body-fixed $(b)$ coordinate system. The latter is chosen as follows: the origin coincides with the hydrodynamic center, the third coordinate axis runs parallel to the threefold symmetry axis of the triskelion, the first and third coordinate axes define the plane that includes the proximal segment of the first leg of the triskelion, and the second coordinate axis lies perpendicular to this plane, in such a way as to form a righthanded coordinate system. In Brownian dynamics, the effect of numerous collisions with solvent molecules is translated into a friction and a random term. The translational motion of the hydrodynamic center over a time step $\delta t$ then reads as

$$
\begin{aligned}
\mathbf{r}_{i}^{(l)}(t+\delta t)= & \mathbf{r}_{i}^{(l)}(t)+\boldsymbol{\mu}^{t(l)}(t) \mathbf{F}_{i}^{(l)}(t) \delta t \\
& +\left[\boldsymbol{\mu}_{i}^{t(l)}(t)\right]^{1 / 2} \boldsymbol{\Theta}_{i}^{t}(t) \sqrt{2 k_{B} T \delta t}
\end{aligned}
$$

where $\boldsymbol{\mu}_{i}^{t(l)}$ denotes the laboratory-based translational mobility tensor, $\mathbf{F}_{i}^{(l)}$ is the sum of all conservative forces acting on the triskelion, and $\boldsymbol{\Theta}_{i}^{t}$ is a random Markovian contribution distributed according to a standard normal distribution. The random displacements are related to the mobility tensor by the fluctuation-dissipation theorem, incorporated by the last term of Eq. (9), where $k_{B}$ is Boltzmann's constant and $T$ temperature. The translational mobility tensor varies with the orientation of the particle,

$$
\boldsymbol{\mu}_{i}^{t(l)}(t)=\mathbf{A}_{i}(t) \boldsymbol{\mu}^{t(b)} \mathbf{A}_{i}^{\mathrm{T}}(t),
$$

where $\mu_{i}^{t(b)}$ represents the constant mobility tensor in the bodyfixed coordinate system. The square-rooted matrix, which obeys $\left[\boldsymbol{\mu}_{i}^{t(l)}\right]^{1 / 2}\left[\boldsymbol{\mu}_{i}^{t(l)}\right]^{1 / 2}=\boldsymbol{\mu}_{i}^{t(l)}$, rotates likewise and hence the matrix square root has to be evaluated only once in the entire simulation.

We have recently introduced a Rotational Brownian Dynamics (RBD) algorithm ${ }^{50}$ to simulate the rotational dynamics of rigid anisotropic bodies by expressing their orientation in space through unit quaternions, i.e., a set of four coordinates $q_{\gamma}$, with $\gamma \in\{0,1,2,3\}$, and a constraint of unit length $|\mathbf{q}|=1$. The rotation matrix $\mathbf{A}$ is then expressed as

$$
\mathbf{A}(\mathbf{q})=\left(\begin{array}{ccc}
q_{0}^{2}+q_{1}^{2}-q_{2}^{2}-q_{3}^{2} & 2\left(q_{1} q_{2}-q_{0} q_{3}\right) & 2\left(q_{1} q_{3}+q_{0} q_{2}\right) \\
2\left(q_{1} q_{2}+q_{0} q_{3}\right) & q_{0}^{2}-q_{1}^{2}+q_{2}^{2}-q_{3}^{2} & 2\left(q_{2} q_{3}-q_{0} q_{1}\right) \\
2\left(q_{1} q_{3}-q_{0} q_{2}\right) & 2\left(q_{2} q_{3}+q_{0} q_{1}\right) & q_{0}^{2}-q_{1}^{2}-q_{2}^{2}+q_{3}^{2}
\end{array}\right) .
$$

This description avoids the well-known singularities encountered when using three angular coordinates, e.g., Euler angles. The resulting algorithm, in the Itô representation, ${ }^{70}$ is numerically stable and remarkably compact, with the $i$ th particle updated by

$$
\begin{aligned}
\mathbf{q}_{i}(t+\delta t)= & \mathbf{q}_{i}(t)+\mathbf{B}_{i}(t) \boldsymbol{\mu}_{i}^{r(b)} \mathbf{A}_{i}^{\mathrm{T}}(t) \mathbf{T}_{i}^{(l)}(t) \delta t \\
& +\mathbf{B}_{i}(t)\left[\boldsymbol{\mu}_{i}^{r(b)}\right]^{1 / 2} \boldsymbol{\Theta}_{i}^{r}(t) \sqrt{2 k_{B} T \delta t}+\lambda_{i}(t) \mathbf{q}_{i}(t),
\end{aligned}
$$

where $\boldsymbol{\mu}_{i}^{r(b)}$ is the constant rotational mobility matrix in the body fixed frame. The second term on the r.h.s. represents the contribution due to the torque $\mathbf{T}_{i}^{(l)}$ acting on the triskelion, and the third term represents the Brownian random contribution, where the components of the vector $\boldsymbol{\Theta}_{i}^{r}$ are again Markovian and distributed according to a standard Gaussian. The transformation matrix $\mathbf{B}_{i}$ converts angular displacements in the body frame into quaternion displacements, ${ }^{69,73}$

$$
\mathbf{B}(\mathbf{q})=\frac{1}{2}\left(\begin{array}{rrr}
-q_{1} & -q_{2} & -q_{3} \\
q_{0} & -q_{3} & q_{2} \\
q_{3} & q_{0} & -q_{1} \\
-q_{2} & q_{1} & q_{0}
\end{array}\right)
$$

In the last contribution to Eq. (12), $\lambda_{i}$ is a Lagrange multiplier used to constrain the modulus of the quaternion to be unitary. Its value is obtained by solving the quadratic equation

$$
\left|\mathbf{q}_{i}^{u}(t+\delta t)+\lambda_{i}(t) \mathbf{q}_{i}(\delta t)\right|^{2}=1
$$

in every step, where $\mathbf{q}_{i}^{u}(t+\delta t)$ are the unconstrained quaternions obtained when $\lambda_{i}=0$. The constraint also conveniently eliminates the calculation of a metric tensor correction and a drift term resulting from the $\mathbf{q}$-dependent mobility, as both turn out to be parallel to the constraint direction. ${ }^{50}$ The translational and rotational mobility tensors are related to their diffusivity counterparts by $\mathbf{D}=k_{B} T \boldsymbol{\mu}$. The latter are determined using the HYDRO++ package $^{72}$ by modeling clathrin as an array of 52 spheres arranged to reflect the overall shape of the protein, using a viscosity of $\eta=10^{-3} \mathrm{~Pa}$ s for water at room temperature. In the body-fixed frame discussed above, the body-fixed translational and rotational diffusivity tensors are both simultaneously diagonal, with $D_{1}^{t}=D_{2}^{t}=1.29 \times 10^{-7} \mathrm{~cm}^{2} \mathrm{~s}^{-1}$ and $D_{3}^{t}=1.07 \times 10^{-7} \mathrm{~cm}^{2} \mathrm{~s}^{-1}$ for the translational diffusivity tensor, and with $D_{1}^{r}=D_{2}^{r}=1.64 \times 10^{4} \mathrm{~s}^{-1}$ and $D_{3}^{r}=1.02 \times 10^{4} \mathrm{~s}^{-1}$ for the rotational diffusivity tensor. The square roots of the 
body-based mobility tensors, as needed in the evaluation of the random terms, are then readily obtained by taking the square roots of the diagonal elements. The time step is set at $10^{-8} \mathrm{~s}$, which is the limit dictated by the rotational Brownian motion of the triskelia.

\section{B. Adaptor protein 2}

\section{The AP2 model}

In a previous study, ${ }^{51}$ we introduced a coarse-grained model for adaptor proteins inspired by the AP2 complex and used it to study their role in the assembly process of clathrin triskelia in bulk through Monte Carlo simulations. Only those parts of the AP2 complex involved in clathrin binding were represented in the model. Here the model is extended to include the core section of the protein, which was excluded in the previous model as it plays no role in the clathrin binding process in bulk. The two clathrin binding sites present in the $\mathrm{C}$-terminal region of the $\beta$ linker, i.e., residues $631-635$ and the $\beta$ appendage domain formed by residues 705-937, are modelled by point particles, $\beta_{1}$ and $\beta_{2}$, respectively, see Fig. 1 . The flexible, structureless linker connecting the two binding sites is composed of about 70 residues, with an estimated contour length of $L_{12} \approx 26 \mathrm{~nm}$. The linker, which acts as an entropic spring, ${ }^{74}$ is modelled through a finite extensible nonlinear elastic (FENE) potential, $^{75}$

$U_{12}(r)=\left\{\begin{array}{cc}-\frac{1}{2} k_{12} L_{12}^{2} \ln \left[1-\left(\frac{r}{L_{12}}\right)^{2}\right] & \text { for } \quad r<L_{12}, \\ \infty & \text { for } r \geq L_{12}\end{array}\right.$

where $r$ denotes the distance between the two beads. The spring constant $k_{12}$ is estimated using an expression from polymer physics, $^{74}$

$$
k_{12}=\frac{3 k_{B} T}{2 L_{12} l_{p}}
$$

where $l_{p}$ denotes the persistence length. With an experimental value of $l_{p} \approx 0.6 \mathrm{~nm}$ for disordered proteins, the resulting spring constant for the linker becomes $k_{12}=30 k_{B} T / \sigma^{2}$.
The core section of the AP protein is responsible for binding with the membrane and cargo. Following the above reductionist approach, it is represented by an additional point particle, $\beta_{0}$, and connected by a flexible tether to the $\beta_{1}$ point particle, see Fig. 1. This section of the linker, representing the initial section of the $\beta$ linker and composed of about 40 residues, has a contour length of $L_{01} \approx 15 \mathrm{~nm}$ and is also modelled through a FENE potential. The spring constant is estimated through Eq. (16) as $k_{01}=50 k_{B} T / \sigma^{2}$. The $\alpha$ linker and $\alpha$ appendage of AP2 are not represented in the model as they do not bind with clathrin nor with the membrane.

\section{Interaction with clathrin}

APs possess two different binding sites for clathrin that specifically bind to two matching sites on a triskelion leg. The presence of a binding site at the end of the TD matching the $\beta_{1}$ site on the AP2 linker is well established. ${ }^{12}$ The AP2 $\beta_{2}$ site binds clathrin near the ankle ${ }^{16}$ and possibly also at a site higher up along the leg. ${ }^{59}$ In previous work, we explored the effect of sites located at the ankle and at the knee on cage assembly in solution, establishing that both possibilities permit AP-regulated clathrin aggregation. ${ }^{51}$ Our study revealed that the mechanical properties of the AP linker region play a crucial role in regulating the assembly mechanism. Here, the $\beta_{2}$ bead binds to the clathrin ankle only, as this is the accepted site in the current literature.

To overcome the numerically inconvenient short range of the AP-clathrin site-site interaction, we developed a clicking potential to describe the AP binding interactions with clathrin. ${ }^{51}$ Consider the interaction between the $\alpha$ th binding site on the $i$ th triskelion and the $\beta$ th bead on the $j$ th AP. Said interaction is described by a flag $b_{i \alpha, j \beta}$ that assumes two values reflecting the discrete state of the interaction. In the unclicked state, $b_{i \alpha, j \beta}=0$, there is no interaction. In the clicked state, $b_{i \alpha, j \beta}=1$, the interaction is characterized by a fixed interaction energy $\epsilon_{\mathrm{CA}}$ and a maximum distance $\rho_{\mathrm{CA}}$ between the two binding sites. The interaction potential then reads as

$$
\phi_{\mathrm{click}}\left(r_{i \alpha, j \beta}, b_{i \alpha, j \beta}\right)=\left\{\begin{array}{cl}
0 & \text { for } \quad b_{i \alpha, j \beta}=0 \\
\left\{\begin{array}{cl}
-\epsilon_{\mathrm{CA}} \text { for } r_{i \alpha, j \beta}<\rho_{\mathrm{CA}} \\
\infty \text { for } r_{i \alpha, j \beta} \geq \rho_{\mathrm{CA}}
\end{array}\right. & \text { for } \quad b_{i \alpha, j \beta}=1 .
\end{array}\right.
$$

The two distinct clathrin-AP bounds, $\beta_{1}$ binding toes and $\beta_{2}$ binding ankles, are probably characterized by different interaction parameters. Since neither interaction free energy has been measured, to the best of our knowledge, we here assume that both bonds are equally strong and explore a range of values for $\epsilon_{\mathrm{CA}}$ in the simulations. The same radius $\rho_{\mathrm{CA}}=0.25 \sigma$ is used to describe both interactions.

The click interactions are turned on and off by an MC procedure. Attempts to modify the interaction status of a randomly selected AP binding site are made at a rate of $r_{\mathrm{CA}}$ attempts per second and per AP bead. A list is made of all $K$ possible binding sites within the interaction radius $\rho_{\mathrm{CA}}$ of that bead, including the current site if applicable. The unclicked state is also considered, as the $0^{\text {th }}$ option. For each of these $K+1$ trial moves, the accompanying energy change $\Delta \phi_{k}^{\text {click }}$ is calculated, which can only assume the values 0 and $\pm \epsilon_{\mathrm{CA}}$, and one of the states is selected with probability

$$
P_{k}=\frac{\exp \left(-\beta \Delta \phi_{k}^{\text {click }}\right)}{\sum_{k^{\prime}=0}^{K} \exp \left(-\beta \Delta \phi_{k^{\prime}}^{\text {click }}\right)}
$$

Note that the coordinates of the particles are left untouched. Excluded volume interactions between AP beads are omitted for reasons of computational efficiency. We note, however, that excluded volume interactions play an important role in preventing multiple AP beads binding to the same clathrin 
site: this is effectively inhibited in the model by making the clicks mutually exclusive, allowing the AP and clathrin sites to partake in only one interaction at a time. We furthermore note that the total volume of all APs bound to a cage is significantly smaller than the interior volume of the cage.

\section{Propagator}

The positions of the three particles composing an AP are updated separately, in a manner that depends on their clicking status. An unclicked bead follows a translational BD equation of motion, see Eq. (9), with an isotropic mobility tensor $\boldsymbol{\mu}_{\beta}^{t}=\mu_{\beta}^{t} \mathbf{I}$, where $\mathbf{I}$ is the identity matrix. The mobility $\mu_{\beta}^{t}$ is estimated through the Stokes-Einstein equation for isolated Brownian spheres,

$$
\mu_{\beta}^{t}=\frac{1}{6 \pi \eta R_{\beta}},
$$

where $\eta$ is the viscosity of the suspending fluid and $R_{\beta}$ the sphere's radius. Assuming a diameter ${ }^{76}$ of $\sim 9 \mathrm{~nm}$ for the $200 \mathrm{kDa}$ AP2 core and a diameter of $\sim 4 \mathrm{~nm}$ for the appendage domain, the mobilities of the $\beta_{0}$ and $\beta_{2}$ beads become $\mu_{0}^{t}$ $=1.2 \times 10^{10} \mathrm{Ns} / \mathrm{m}$ and $\mu_{2}^{t}=2.6 \times 10^{10} \mathrm{Ns} / \mathrm{m}$, respectively, in water at room temperature. Unlike those two bulky sites, the motion of the four-residue $\beta_{1}$ site will be dominated by the dynamics of the linker. For reasons of simplicity, the $\beta_{1}$ bead is simulated as a Brownian particle with the same properties as the $\beta_{2}$ bead. The beads do not rotate.

We now turn to clicked beads. All AP beads clicked to a triskelion move with that triskelion; the aggregate of a triskelion and one or several attached AP beads moves as a single rigid body, obeying Eqs. (9) and (12), subject to the sum of all the forces and torques acting on the aggregate. This preserves the clicking status of the AP beads involved, as well as their relative positions in the body frame of the triskelion. The translational and rotational mobilities of the aggregate are assumed to be identical to those of a free triskelion. On top of this motion, the AP beads explore the small clicking volume by Monte Carlo trial moves taking them to random positions within this volume, at a rate of $r_{v}$ attempts per second and per bead. These trial moves also preserve the clicking status but alter the potential energy. Trial moves are accepted or rejected with a probability given by the Metropolis scheme ${ }^{77,78}$

$$
P_{o \rightarrow n}^{\mathrm{acc}}=\min \left\{1, e^{-\beta \Delta \Phi_{o \rightarrow n}}\right\},
$$

where $\Delta \Phi_{o \rightarrow n}=\Phi(n)-\Phi(o)$ is the potential energy change between the old configuration $o$ and the new configuration $n$.

\section{Chemostat}

In the relatively small volume of the simulation box, the adhesion of APs and triskelia to the membrane will strongly deplete their concentrations in the dissolved phase. To counter this effect, a chemostat is employed to maintain a constant chemical potential, and hence constant concentration in the bulk phase, by the insertion and removal of particles, thus mimicking exchange with a large ideal reservoir. A Monte Carlo algorithm is applied to both solute molecule types independently; in the following, we refer to clathrin as an illustrative example. Trial moves to exchange triskelia with the reservoir are attempted at a rate of $r_{c}$ attempts per second. The trial move consists in adding or removing a triskelion with equal likelihood. Consider a system with $N_{\mathrm{C}}$ triskelia in a volume $V$. The probability of acceptance of an insertion move is given by 69,78

$$
P_{N_{\mathrm{C}} \rightarrow N_{\mathrm{C}}+1}^{\mathrm{acc}}=\min \left\{1, \frac{[\mathrm{C}]_{r} V}{N_{\mathrm{C}}+1} e^{-\beta \Delta \Phi_{N_{\mathrm{C}} \rightarrow N_{\mathrm{C}}+1}}\right\},
$$

where $[\mathrm{C}]_{r}$ refers to the clathrin concentration in the reservoir and $\Delta \Phi_{N_{\mathrm{C}} \rightarrow N_{\mathrm{C}}+1}$ is the potential energy change accompanying the insertion of a triskelion at a random position and with a random orientation. Similarly, the acceptance probability for the removal of a randomly selected triskelion is given by

$$
P_{N_{\mathrm{C}} \rightarrow N_{\mathrm{C}}-1}^{\mathrm{acc}}=\min \left\{1, \frac{N_{\mathrm{C}}}{[\mathrm{C}]_{r} V} e^{-\beta \Delta \Phi_{N_{\mathrm{C}} \rightarrow N_{\mathrm{C}}-1}}\right\} .
$$

Similar equations apply for the AP2 chemostat.

The chemostat algorithm causes the numbers of triskelia and APs to fluctuate throughout a simulation. For an ideal gas at constant chemical potential, volume, and temperature, the equilibrium distribution of the number of molecules follows a Poisson distribution. A test simulation was run using reference concentrations of $[\mathrm{C}]_{r}=0.01 \sigma^{-3}$ and $[\mathrm{A}]_{r}=0.01 \sigma^{-3}$, respectively, with all inter-molecular interactions turned off. Under these conditions, the system should behave like a mixture of two ideal gases featuring different internal degrees of freedom. Good numerical agreement was observed between the averages and variances of the number of molecules present and their theoretical values (data not shown).

\section{Membrane}

\section{The membrane model}

The free energy of a membrane is well described by the Helfrich expression, ${ }^{74,79}$

$$
F=2 \kappa \int_{A} H^{2} d a+\frac{1}{2} K_{A} A_{0}\left(\frac{A-A_{0}}{A_{0}}\right)^{2},
$$

where the integral runs over the area $A$ of the membrane, $H$ denotes the local mean curvature, $\kappa$ the bending rigidity, $K_{A}$ is the elasticity modulus, and $A_{0}$ is the equilibrium surface area. In this form, the equation applies to membranes with no intrinsic curvature, fixed topology, and without edges. The coarse grained membrane model aims at reproducing Helfrich's free energy.

We simulate the membrane through a mesh composed of $N_{m}$ beads, connected by $3 N_{m}$ flexible tethers forming a multifaceted surface composed by $3 N_{m} / 2$ triangles. The elasticity is accounted for by modeling the flexible tethers through a FENE potential,

$$
U_{m}^{\mathrm{el}}(r)=\left\{\begin{array}{cc}
-\frac{1}{2} k_{m} L_{m}^{2} \ln \left[1-\left(\frac{r-r_{m}^{0}}{L_{m}}\right)^{2}\right] & \text { for }\left|r-r_{m}^{0}\right|<L_{m}, \\
\infty & \text { for }\left|r-r_{m}^{0}\right| \geq L_{m}
\end{array}\right.
$$

where $k_{m}$ denotes the spring constant, and $L_{m}$ the maximum deviation from the equilibrium length $r_{m}^{0}$. Self-avoidance of the membrane is guaranteed by introducing excluded volume interactions between beads, modelled through a WeeksChandler-Andersen (WCA) potential, 


$$
U_{m}^{\mathrm{ev}}(r)=\left\{\begin{array}{cl}
4 \epsilon_{m}^{\mathrm{ev}}\left[\left(\frac{\sigma_{m}^{\mathrm{ev}}}{r}\right)^{12}-\left(\frac{\sigma_{m}^{\mathrm{ev}}}{r}\right)^{6}+\frac{1}{4}\right] & \text { for } \quad r<2^{1 / 6} \sigma_{m}^{\mathrm{ev}}, \\
0 & \text { for } \quad r \geq 2^{1 / 6} \sigma_{m}^{\mathrm{ev}}
\end{array}\right.
$$

The radius $\sigma_{m}^{\mathrm{ev}}=0.35 \sigma$ and strength $\epsilon_{m}^{\mathrm{ev}}=10 k_{B} T$ are chosen such that membrane beads are prevented from crossing the surface of any membrane triangle.

A bending potential is introduced between neighbouring triangles. Several potentials have been proposed in the literature in which the mean curvature is calculated either through the angles formed by adjacent triangles ${ }^{79-81}$ or using a lattice dual to the membrane triangulation. ${ }^{79,81-83}$ The latter approach, unlike the former, yields a bending rigidity that does not vary with the membrane topology ${ }^{81,84}$ and is therefore followed here. For computational convenience, we use a simplified version of this potential, ${ }^{82}$

$$
\Phi=\frac{1}{2} k_{\kappa} \sum_{i} \phi_{i}
$$

where $k_{\kappa}$ denotes the strength of the potential. The summation runs over all membrane beads, with

$$
\phi_{i}=\frac{1}{\Omega_{i}}\left[\sum_{j(i)}\left(\mathbf{r}_{i}-\mathbf{r}_{j}\right)\right]^{2},
$$

where the sum runs over all nodes $j$ connected to $i$ and $\Omega_{i}$ represents the collective area of all triangles joining at site $i$.

\section{Propagator}

The motion of individual membrane nodes is described by a translational BD equation of motion, see Eq. (9), with a constant isotropic mobility tensor $\boldsymbol{\mu}_{m}^{t}=\mu_{m}^{t} I$. Membrane thermal undulations with wavelengths comparable to the clathrin size relax much faster than the time required for clathrin to diffuse over its own length. ${ }^{85}$ Since we want to simulate both processes, we use the expedient of slowing down the membrane motion by judiciously choosing the highest mobility scalar $\mu_{m}^{t}$ that maintains a numerically stable membrane when using the maximum time step suitable for the triskelia, thus arriving at a diffusion coefficient of $D_{m}^{t}=\mu_{m}^{t} k_{B} T=100 \sigma^{2} / \mathrm{s}$.

Networks with fixed connectivity behave as elastic struts and prove unable to form pit-like invaginations under appropriate forces. To model the fluidity and deformability of the membrane, Monte Carlo moves are used to modify the network connectivity. ${ }^{79}$ A random bond is selected and flipped to connect the tops of the two triangles that hitherto shared this bond, as illustrated in Fig. 3. This move leaves the positions of the nodes untouched, but nevertheless it is likely to result in a change of the potential energy of the flipping bond and of the bending energies of the neighbouring triangles. The acceptance probability of the flipping move is again given by the Metropolis scheme in Eq. (20). Note that the total number of triangles is conserved, while the connectivity numbers of the four nodes involved in the bond swap increase or decrease by one. In order to conserve the geometrical properties of the triangulated network, any attempt that reduces the connectivity of a node below five is rejected. ${ }^{79}$ Bond flips are attempted at a rate of $r_{f}$ attempts per bond and per second.

\section{Interaction with other particles}

The membrane-with a main orientation parallel to the $x y$ plane-floats roughly in the middle of the simulation box and thereby divides the box into an "interior" region above the membrane and an "exterior" region below the membrane (where we, for the sake of argument, temporarily ignore the periodic boundary conditions along the $z$ axis). The presence of particles only in the interior region will result in an osmotic pressure that pushes the membrane down; this effect is removed by employing periodic boundary conditions in the $z$ direction because this allows the particles to diffuse freely between the two regions and thereby equalizes pressures and chemical potentials on both sides of the membrane. A flag $l_{i}$ is assigned to every triskelion and AP molecule to distinguish between those in the interior, $l_{i}=+1$, and those in the exterior, $l_{i}=-1$. When a particle $i$ crosses the periodic boundaries at $z= \pm \frac{1}{2} L_{z}$, its flag $l_{i}$ reverses sign.

The interaction of AP beads with the membrane comprises two parts, a repulsion that prevents the $\beta_{1}$ and $\beta_{2}$ beads from crossing the membrane and a click potential that enables the core, i.e., $\beta_{0}$, to bind to a membrane bead. The repulsive beadmembrane potential should allow particles to come close to the membrane but not to cross the membrane permanently, a combination that cannot be realized in BD simulations by merely introducing a repulsive potential between beads and membrane nodes. Instead, we introduce an interaction between particle $i$ and membrane triangles $t$ of the form

$$
\phi_{i}^{\mathrm{m}}=\epsilon_{\mathrm{m}} \sum_{t} F\left(l_{i} r_{i t}^{\perp}\right) G\left(r_{i t}^{\prime \prime}\right),
$$

where $\epsilon_{\mathrm{m}}$ is a positive strength parameter. Using $\hat{\mathbf{n}}_{t}$ as the normal to the triangle, pointing to the interior, one may calculate the height of the particle above the membrane, $r_{i t}^{\perp}=\mathbf{r}_{i t} \cdot \hat{\mathbf{n}}_{t}$, and its lateral displacement along the membrane, $r_{i t}^{\| l}=\left|\mathbf{r}_{i t}-r_{i t}^{\perp} \hat{\mathbf{n}}_{t}\right|$, with $\mathbf{r}_{i t}$ being the vector pointing from the center of mass of the triangle to the bead. The above introduced flag $l_{i}$ is included in the argument of $F$ to ensure that the resulting repulsive force always points in the correct direction, i.e., to the interior for $l_{i}$ $=+1$ and to the exterior for $l_{i}=-1$, even if the bead has slightly penetrated the membrane.

The penalty function $F$ is zero for particles beyond a height $x_{F}$, is quadratic just above the membrane triangle, and

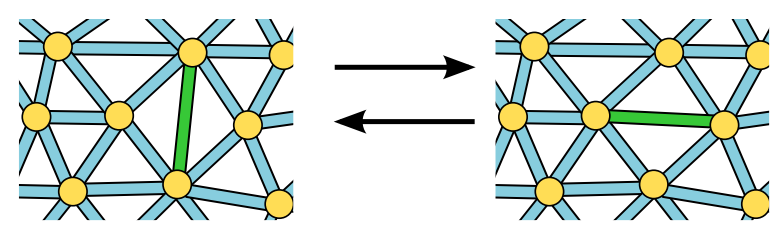

FIG. 3. Cartoon of the Monte Carlo bond flipping move, employed in the simulations to ensure membrane fluidity and enable large amplitude deformations. A randomly selected bond, here coloured in green, is flipped from one diagonal to the other diagonal of the quadrangle formed by its two adjacent triangles. 
increases linearly for particles penetrating the membrane,

$$
F(x)=\left\{\begin{array}{clr}
0 & \text { for } & x \geq x_{F}, \\
\left(x-x_{F}\right)^{2} & \text { for } & 0<x<x_{F}, \\
x_{F}^{2}-2 x_{F} x & \text { for } & 0 \leq x
\end{array}\right.
$$

The function $G$ distributes the interaction over several adjacent triangles, reflecting the size of the protein fragment interacting with the membrane,

$$
G(x)=\left\{\begin{array}{clr}
\left(x-x_{G}\right)^{2} & \text { for } & 0<x<x_{G} \\
0 & \text { for } & x \geq x_{G}
\end{array}\right.
$$

with $x_{G}$ setting the decay length of the spread function. In the simulations, $\epsilon_{\mathrm{m}}=10^{3} k_{B} T / \sigma^{4}, x_{F}=0.1 \sigma$, and $x_{G}=1 \sigma$.

The above repulsion applies to the $\beta_{1}$ and $\beta_{2}$ beads of AP, as well as to the hubs, knees, and ankles of triskelia. The core particle of AP is excluded to permit binding of this particle to the membrane, as discussed below. A modest flexibility of the clathrin protein, allowing a leg to bend when pressed against a membrane, is mimicked in the simulations by a reduction by $90 \%$ of the repulsion strength $\epsilon_{\mathrm{m}}$ for the extremal parts of the triskelion, i.e., the ends of the TDs.

The short-ranged binding interaction between the AP core, $\beta_{0}$, and a membrane node is described, like the equally short-ranged AP-clathrin binding interaction, by the clicking potential introduced in Section II B 2. Attempts are made, at a rate of $r_{\mathrm{A} m}$ attempts per AP and per second, to click a free core with any available membrane node within a clicking radius of $\rho_{\mathrm{A} m}=0.1 \sigma$ or to unclick a bound core. Upon clicking (unclicking), the system energy is lowered (raised) by the click energy $\epsilon_{\mathrm{A} m}$. Clicks are again mutually exclusive, to prevent two APs from binding to the same membrane bead. Structural studies have revealed that the AP2 protein undergoes a large conformational change from a "locked" state in the cytosol to an "open" state when bound to the membrane. ${ }^{14,15}$ In the locked state, the $\beta_{1}$ site is buried within the core and only the $\beta_{2}$ site is available for clathrin binding, thus inhibiting APs from bridging two triskelia and thereby effectively suppressing the cage assembly process in the cytosol. Upon binding to the membrane, the $\beta_{1}$ site is released and the AP2 complex can bind two triskelia. This mechanism is mimicked in the simulations by allowing a $\beta_{1}$ bead to click to the end of a TD only when the matching core bead is clicked to a membrane node. Since a membrane-bound AP2 complex can diffuse relative to the membrane, in the simulation MC moves are included that enable the AP core bead to jump from its membrane node to a randomly selected neighbouring node, at a rate of $r_{j}$ attempts per membrane bound AP and per second. The acceptance probability of trial moves to unoccupied nodes is again given by Eq. (20), while trial jumps to occupied nodes are rejected.

\section{Monte Carlo barostat}

Together with the bending rigidity, tension is a key factor determining the membrane deformability. A change of the in-plane tension can be induced by an affine rescaling of the simulation box, thus stretching or compressing all tethers forming the triangulated mesh. A vanishing tension is imposed on the system through Monte Carlo rescaling moves affecting the two in-plane directions $x$ and $y$ independently, while preserving the size of the box in the $z$ direction. Attempts are made at a rate of $r_{b}$ per second to change the length of a selected box edge from the old value $L_{o}$ to a new value $L_{n}=L_{o}+\Delta L$, where $\Delta L$ is a random number uniformly distributed over the interval $[-0.1,0.1] \sigma$. Rescaling causes a volume change of the simulation box from $V_{o}$ to $V_{n}$. The affine transformation is applied to all membrane beads, the hydrodynamic centers of the triskelia, and all unclicked AP beads; clicked AP beads move with the bead they are connected to, in order to preserve their click statuses. The probability of accepting the trial move as the new configuration is given by $^{78}$

$$
P_{o \rightarrow n}^{\mathrm{acc}}=\min \left\{1,\left(\frac{L_{n}}{L_{o}}\right)^{N} e^{-\beta\left[\Delta \phi_{o \rightarrow n}+P\left(V_{n}-V_{o}\right)\right]}\right\},
$$

where $N$ is the total number of molecules in the systemincluding the membrane, counted as one molecule - and $P$ the imposed pressure. To obtain a tensionless membrane, $P$ is set to zero. Since the two dimensions are scaled independently, the initial shape of the box is not conserved throughout the simulation.

\section{Model validation}

The mechanical properties of the membrane were tested using a system containing 1188 membrane nodes, initially prepared in a regular hexagonal lattice. The equilibrium length of the tethers was set at $r_{m}^{0}=0.5 \sigma$ and the maximum extensibility at $L_{m}=0.8 \sigma$. Typical experimental values for giant unilamellar vesicles of fluid-phase double-tailed phosphatidylcholine lipids at room temperature were selected for the bending rigidity, $\kappa=0.4 \times 10^{-19} \mathrm{~J}=10 k_{B} T$, and the elastic modulus, $K_{A}=250 \mathrm{~mJ} / \mathrm{m}^{2} ;{ }^{86}$ the former is realised in the simulation model by $k_{\kappa}=\kappa$, the latter through the formula $k_{m}=2 K_{A} / \sqrt{3}$ obtained by relating area expansion to tether elongation in a hexagonal lattice. During simulations with this membrane, the MC steps to swap bonds were never accepted and consequently the membrane displayed anisotropic behaviour. Moreover, when initiating simulations with a membrane containing a hand-made pit-like dome, which required the introduction of beads with 5 or 7 neighbours, the pit was never observed to collapse spontaneously. Since the main properties of a membrane relevant for the endocytic process are its non-permeability and bending stiffness, we found it expedient to lower the elastic modulus to $1 \mathrm{~mJ} / \mathrm{m}^{2} \approx 70 k_{B} T / \sigma^{2}$. The resulting model membrane permits bond swaps and shows isotropic behaviour, and artificially introduced domes readily collapse.

The elastic modulus of the model membrane was determined by a series of runs uniformly stretching both in-plane box dimensions from $L_{\|}=220 \sigma$ to $230 \sigma$, with the barostat turned off. The tension on the membrane $\tau_{\|}$was calculated from the diagonal elements of the stress tensor, $P_{\alpha \beta}$, where the latter was obtained by the virial expression. ${ }^{69,78}$ When the elastic contribution dominates the Helfrich free energy, the tension is related to the relative elongation of the membrane by $^{87,88}$

$$
\tau_{\|}=L_{z}\left[P_{z z}-\frac{1}{2}\left(P_{x x}+P_{y y}\right)\right]=K_{A \| 0} \frac{A_{\|}-A_{\| 0}}{A_{\| 0}},
$$


where the membrane area is approximated by the ground plane area of the simulation box, $A_{\|}=L_{\|}^{2}$. The stress-strain curve of the membrane is shown in Fig. 4. From the slope of the tangent at the zero-tension intercept, at $A_{\| 0} \approx 224.4 \sigma^{2}$, we obtain an effective elastic modulus $K_{A \| 0} \approx 58 k_{B} T / \sigma^{2}$. The curve is not the straight line predicted by Eq. (32), and the elastic modulus is less than expected, because the contributions of the membrane undulations to both the free energy and the membrane area are ignored in that equation. Both can be corrected for, yielding $86,88,89$

$$
K_{A \| 0}^{-1}=K_{A}^{-1}+\frac{k_{B} T}{32 \pi^{3} \kappa^{2}} A_{\| 0} .
$$

Upon inserting the bending rigidity (to be discussed next), we obtain $K_{A}=67 k_{B} T / \sigma^{2}$, in good agreement with the input value. This value is also approached by the slope of the curve in the large area limit of Fig. 4, where the high tension has effectively suppressed the undulations. Upon turning the barostat on, the areas of the simulation boxes were observed to converge to $A_{\| 0}$.

The undulations of the membrane are commonly expressed by a superposition of two-dimensional transverse waves. Taylor expanding the Helfrich free energy to second order in the complex amplitudes $c_{\mathbf{q}}$ of a Fourier series with wave vectors $\mathbf{q}$, followed by applying the equipartition theorem, yields the structure factors 87,90

$$
S(\mathbf{q})=\left\langle\left|c_{\mathbf{q}}\right|^{2}\right\rangle=\frac{k_{B} T}{A_{\|}\left(\kappa q^{4}+\tau q^{2}\right)},
$$

where $\tau$ is the tension on the membrane. Figure 5 shows the results for two barostatted membranes with $k_{\kappa}=10 k_{B} T$ and $20 k_{B} T$, respectively. From the slopes of the fitted straight lines, we extract effective bending rigidities of $\kappa=11 k_{B} T$ and $18 k_{B} T$, respectively, in good agreement with the input values. The small non-zero intercept indicates that there is a small residual tension on the membrane, which arises from a subtle

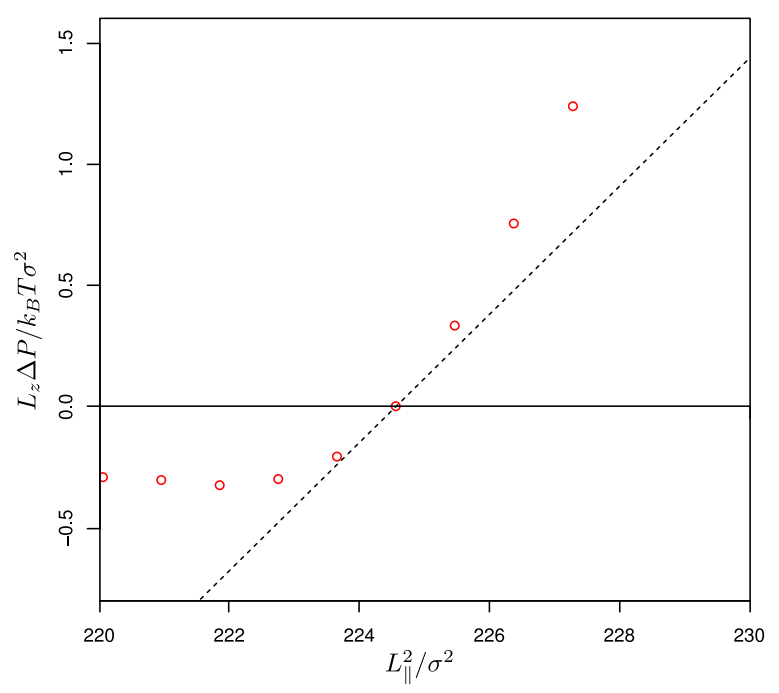

FIG. 4. The tension on the membrane as a function of the area of the ground plane of the simulation box. The non-linearity of the curve reflects the presence of membrane undulations: undulations are suppressed under elongation and promoted under compression, causing the membrane to buckle for the smallest areas. The slope of the dotted line, i.e., the tangent at the tensionless state, yields the effective elastic modulus $K_{A \| 0}$.

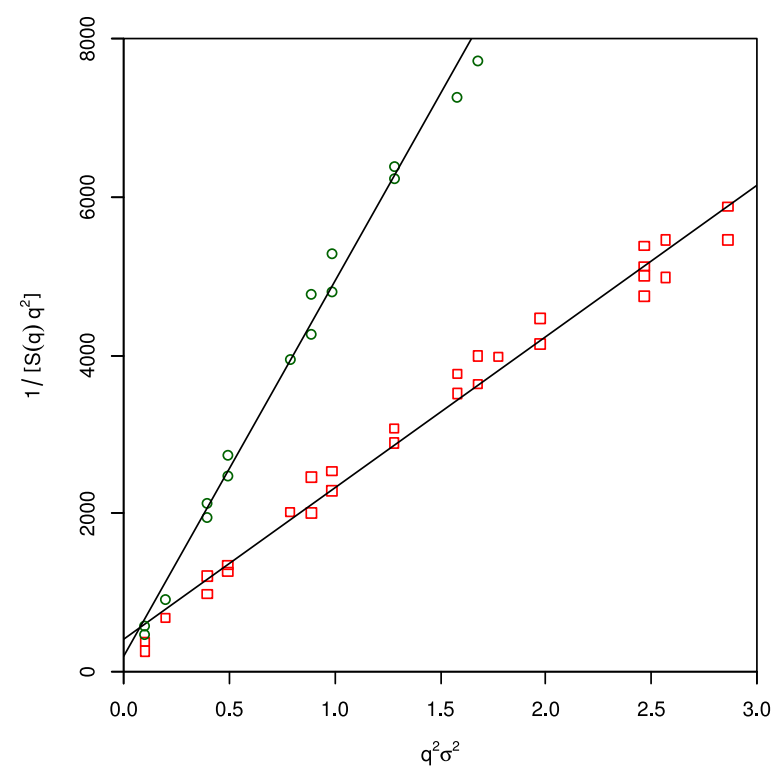

FIG. 5. Structure factors of thermal membrane undulations $S$ versus wave number $q$, plotted in a format that linearizes their theoretical relationship by Eq. (34). The green circles and red squares mark simulations at two differing input values of the bending rigidity $k_{\kappa}$.

difference between the tension $\tau$ measured in this plot and the tension $\tau_{\|}$nullified by the barostat. ${ }^{88,91}$

\section{RESULTS}

\section{A. Membrane coverage}

The beads of our membrane model perform three roles. First, the positions of the beads describe the configuration of the membrane and the interactions between the beads endow the membrane with an equilibrium area, an elastic modulus, and a bending rigidity. Second, the multi-faceted plane formed by the beads acts as a barrier that prevents molecules from crossing the membrane. Third, the membrane beads act as binding sites for APs, in the same way that the combination of PtdIns $(4,5) \mathrm{P}_{2}$ and cargo functions as the membranebound binding partner for the AP2 complex in vivo. The latter role is the focus of the current subsection. AP2 is modelled as a chain of three beads connected by springs, see Fig. 1. The first bead, $\beta_{0}$, represents the core and can bind to a membrane bead. Beads $\beta_{1}$ and $\beta_{2}$ along the $\beta$ linker are capable of binding to the clathrin terminal domain and knee, respectively.

When the membrane is exposed to a solution of APs, a number of membrane beads will become occupied by one APcore bead each. The fractional occupancy $\theta_{\mathrm{A}}$ is expected to be a function of the bulk AP concentration, [A], and the strength of the binding interaction, $\epsilon_{\mathrm{A} m}$. This relation was explored by varying the bond strength in a series of simulations using a membrane of 2064 nodes. A chemostat simulates the exchange of APs with a hypothetical ideal AP reservoir and thereby maintains a bulk AP concentration of $0.01 \sigma^{-3}$, irrespective of the number of APs attached to the membrane. To minimize interference with the processes at the membrane and to avoid possible superpositions of inserted particles with the membrane surface, the chemostat is applied only to a section of 
the box well below the membrane, i.e., in the "exterior" to the cell, covering $\sim 25 \%$ of the total box volume. Diffusion of molecules between the "exterior" and "interior," via the periodic boundary conditions along the $z$ direction, equalizes the chemical potential and osmotic pressure throughout the box.

To accelerate the realization of thermodynamic equilibrium and to improve sampling, in this subsection only, the membrane is configured as an immobile ideal hexagonal lattice, the barostat is turned off, and APs are not permitted to jump diffusively between membrane beads-note that these expedients do not affect the equilibrium occupancy. We remind the reader that the interaction between membrane-clicked APs is designed to prevent multiple APs from binding to the same membrane node, thus mimicking explicit excluded volume interactions that are omitted for reasons of computational efficiency. Clathrin triskelia are absent in this initial set of simulations.

The simulation results collected in Fig. 6 show a smooth transition, from a low occupancy of the membrane beads to their near saturation with APs, in a narrow region of clicking strengths $\epsilon_{\mathrm{A} m}$. A statistical-mechanical derivation of this Langmuir-like behaviour ${ }^{93}$ is presented in the Appendix, yielding

$$
\frac{\theta_{\mathrm{A}}}{1-\theta_{\mathrm{A}}}=\frac{[\mathrm{A}]}{c_{0}} e^{-\beta \Delta G_{\mathrm{A} m}^{0},}
$$

where $c_{0}$ denotes the standard reference concentration of $1 \mathrm{M}$, and $\Delta G_{\mathrm{A} m}^{0}$ is the change in the standard state free energy accompanying the AP-membrane binding reaction. That is,

$$
\Delta G_{\mathrm{A} m}^{0}=\mu_{\mathrm{A} m}^{0}-\mu_{\mathrm{A}}^{0}-\mu_{m}^{0}=\Delta \mu_{\mathrm{A} m}^{0},
$$

with $\mu_{\mathrm{X}}^{0}$ the reference chemical potential of component $\mathrm{X}$ at the standard concentration and $\Delta \mu_{\mathrm{A} m}^{0}$ the standard chemical

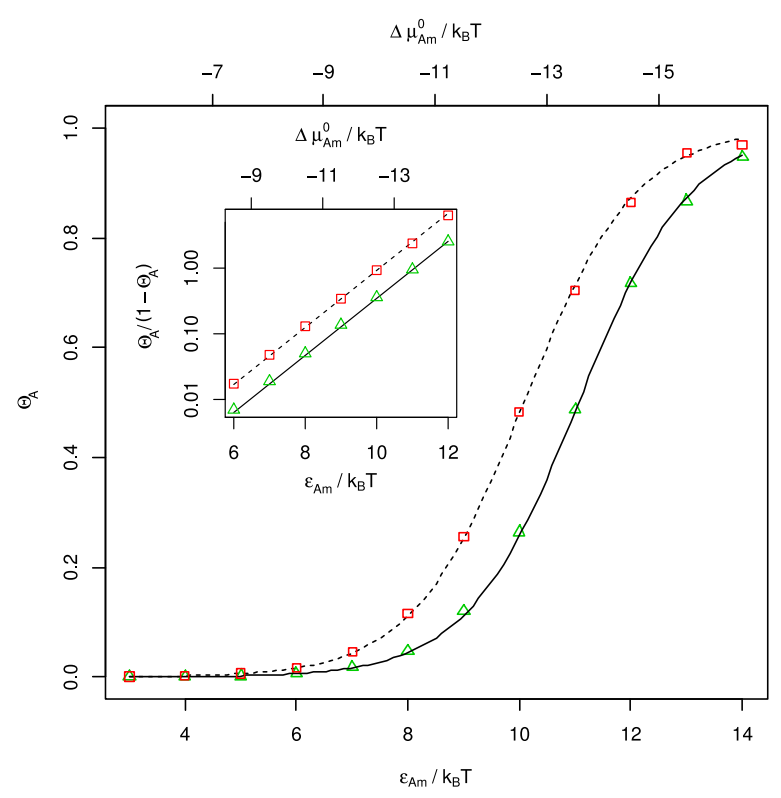

FIG. 6. The fraction $\theta_{\mathrm{A}}$ of membrane beads occupied by AP as a function of the AP-membrane binding strength $\epsilon_{\mathrm{A} m}$, for a membrane exposed to an AP solution of $[\mathrm{A}]=10^{-2} \sigma^{-3} \approx 3.4 \times 10^{-6} \mathrm{M}$, with the restriction potential preventing AP's linker beads $\beta_{1}$ and $\beta_{2}$ from crossing the membrane turned off (red squares, dashed line) and turned on (green triangles, solid line). Markers represent averages over simulations of $10 \mathrm{~s}$ each; the lines are obtained by the statistical-mechanical theory outlined in the Appendix. The inset shows a linear representation of the same data. potential difference associated with the reaction. The agreement between the theory and simulations is very good, see Fig. 6 , both in the absence and presence of the potential that prevents the two AP linker beads $\beta_{1}$ and $\beta_{2}$ from crossing the membrane. The reduced configurational freedom imposed by this restraint causes a shift of the equilibrium fractional coverage function to slightly higher binding energies. Chang et al. ${ }^{92}$ measured the saturation binding of AP2 on plasma membrane fragments prepared by freeze-thaw lysis of cells, which were stripped of their endogenous coat proteins before exposure for 30 min to AP2 solutions of various concentrations. Their experimental data are well described by the Langmuir theory, see Fig. 7, yielding $\Delta \mu_{\mathrm{A} m}^{0}=-17.8 k_{B} T$.

Let us now consider a mixture of clathrin and APs, both subject to a chemostat. As in our preceding simulation studies, ${ }^{48,49}$ clathrin is modelled as a rigid triskelion with kinked legs following the characteristic shape of the protein, see Fig. 1. Attractions (repulsions) are introduced between pairings of straight leg segments that are (are not) present in experimental cage edges. The interactions were originally tuned to simulate in vitro self-assembly in a slightly acidic solution; upon weakening these interactions, the model reproduces the in vitro regulation of cage assembly by AP $2 .{ }^{51}$ The AP 2 complex carries a mid-linker clathrin binding site that is available only when AP2 binds to PtdIns(4,5) $\mathrm{P}_{2}$ and cargo at a membrane. ${ }^{15}$ This feature is mimicked in the AP simulation model by activating the clicking potential of the $\beta_{1}$ site of an AP molecule only when its core bead is clicked to a membrane bead. With the membrane bead representing a patch of membrane including PtdIns $(4,5) \mathrm{P}_{2}$ and cargo, the $\beta_{1}$ sites of all membrane-bound APs are activated. The proper functioning of this feature is tested by temporarily simplifying the properties of the AP model to the point where the membrane coverage can be solved analytically. In this simplified model, the $\beta_{2}$ bead does not bind clathrin, the number of AP binding sites per clathrin is reduced

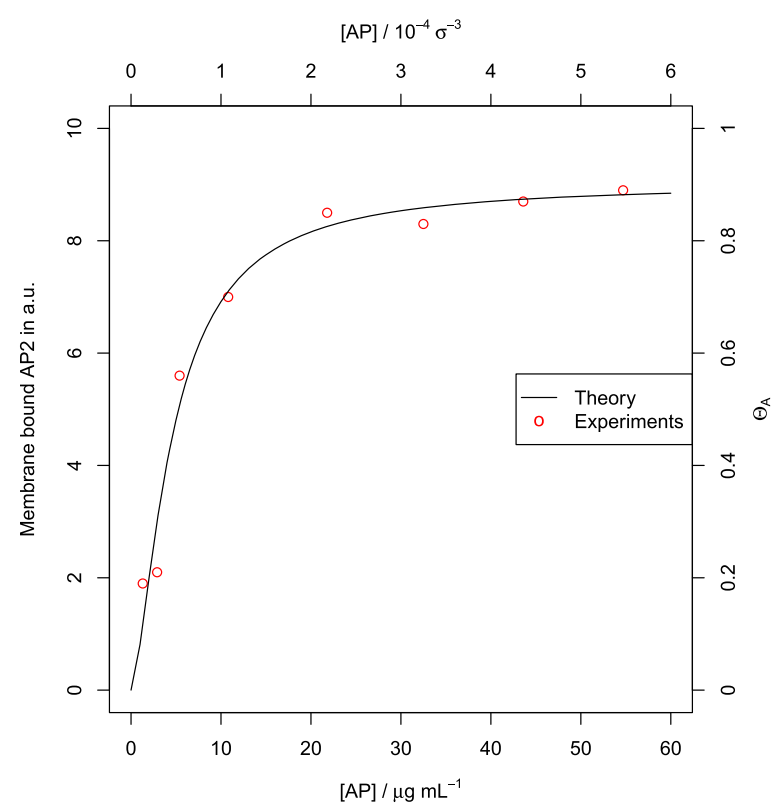

FIG. 7. The coverage of a membrane by AP2 as a function of the AP2 concentration in the bulk, in arbitrary units (a.u.). Markers show experimental data by Chang et al. ${ }^{92}$ the solid line is a fit with the Langmuir theory, see Eq. (35). 


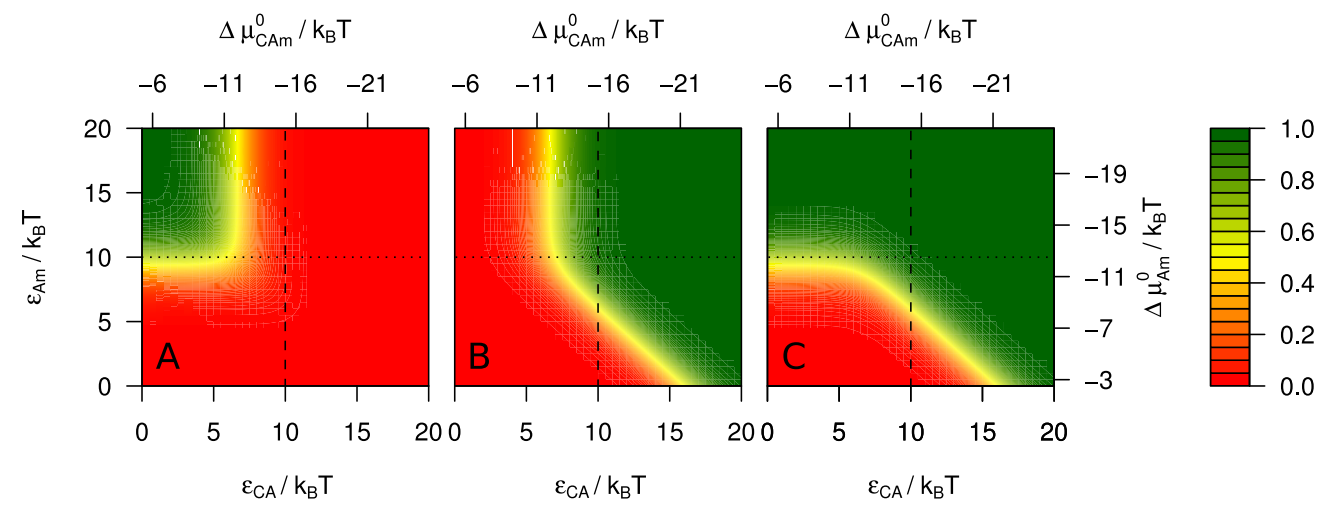

FIG. 8. The theoretical fractional occupancies $\theta$ of membrane beads (a) by AP, $\theta_{\mathrm{A}}$, (b) by AP-clathrin complexes, $\theta_{\mathrm{CA}}$, and (c) the sum of these two occupancies. In order to make this theoretical calculation feasible, AP has been curtailed to one clathrin binding site, which is activated only when AP is bound to the membrane, the triskelia have only one site that clicks to AP, and the penetration barrier of the membrane is turned off. The green area below the dotted line in the two rightmost plots, while the leftmost plot is red at these low membrane-AP binding strengths, highlights a cooperative effect with APs binding to the membrane only in the presence of triskelia. The concentrations of free monomers in the solution are fixed at $[\mathrm{A}]=10^{-2} \sigma^{-3}$ and $[\mathrm{C}]=10^{-3} \sigma^{-3}$. Simulation results along the dashed and dotted lines are presented in Fig. 9.

to one, at the end of one leg, and the interactions among clathrin triskelia are turned off.

A statistical mechanical derivation of the fractional occupations of membrane sites by APs and AP-clathrin complexes is presented in the Appendix, and the resulting phase diagrams are depicted in Fig. 8. In agreement with the simulations discussed above, Fig. 8(a) shows that APs saturate the membrane beads when the standard chemical potential difference associated with AP clicking to the membrane, $\Delta \mu_{\mathrm{A} m}^{0}$, is lower (more negative) than about $-12 k_{B} T$. Below this value, i.e., for the high binding strengths $\epsilon_{\mathrm{A} m}$ at the top of the plots, with increasing $\mathrm{AP}$-clathrin binding strength, $\epsilon_{\mathrm{CA}}$, a transition from predominantly unoccupied APs to APs occupied by triskelia is observed in Figs. 8(a) and 8(b) when the standard chemical potential difference associated with clathrin clicking to a membrane-bound AP reaches $\Delta \mu_{\mathrm{CA} m}^{0} \approx-12 k_{B} T$. Simulations with the simplified coarse-grained models, employing the acceleration expedients listed above, yield good agreement with the theory, see Fig. 9(a). Because this standard chemical potential difference is closely related to that of clathrin binding a (hypothetical) active $\beta_{1}$-bead of an AP in solution,

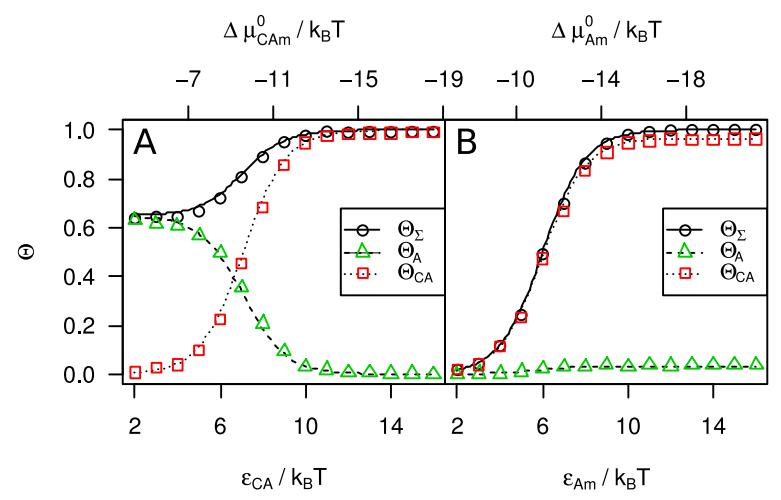

FIG. 9. The simulated fractional occupancies $\theta$ of membrane beads by AP $\left(\theta_{\mathrm{A}}\right.$, green triangles), by AP-clathrin complexes ( $\theta_{\mathrm{CA}}$, red squares), and their sum (black circles), for (a) an AP-membrane binding strength $\epsilon_{\mathrm{A} m}=10 k_{B} T$, and (b) an AP-clathrin binding strength $\epsilon_{\mathrm{CA}}=10 k_{B} T$, The lines represent the corresponding theoretical curves, i.e., the cross sections of Figs. 8(a)-8(c) along the dotted and dashed lines, respectively.
$\Delta \mu_{\mathrm{CA} m}^{0} \approx \Delta \mu_{\mathrm{CA}}^{0}$, the onset of AP-assisted clathrin adsorption at the membrane approximately coincides with the onset of AP-clathrin complexation in the bulk if the latter is not prevented by AP adopting the closed conformation in solution. Interestingly, the triangular green region for $\Delta \mu_{\mathrm{A} m}^{0} \approx-12 k_{B} T$ in Figs. 8(b) and 8(c), while Figs. 6 and 8(a) show that $\theta_{\mathrm{A}} \approx 0$ for $\Delta \mu_{\mathrm{A} m}^{0} \approx-12 k_{B} T$, indicates that the binding of AP to the membrane can be strongly affected by clathrin: for the combination of binding strengths corresponding to the green triangular area, the AP-membrane interaction is too weak to induce AP adsorption, but the (prospective) release of the APclathrin binding free energy - which requires AP binding to the membrane-makes the APs click to the membrane nevertheless. This cooperative behaviour is confirmed by the simulation results presented in Fig. 9(b). If endocytosis is initiated by AP2 tethering clathrin to the plasma membrane in response to the detection of cargo molecules, then this sequence of events will occur in the current simplified model if the combination of AP-membrane and AP-clathrin interactions corresponds to a point in the red area of Fig. 8(b) in the absence of cargo and shifts upwards-increasing the strength of the AP-membrane bond-into the green region of that figure in the presence of cargo.

\section{B. Formation of coated pits}

The aggregation of triskelia at the membrane was studied using the full simulation model described in Section II. A chemostat stabilized the bulk concentrations of clathrin and $\mathrm{AP}$ at $[\mathrm{C}]=10^{-3} \sigma^{-3}$ and $[\mathrm{A}]=10^{-2} \sigma^{-3}$, respectively. The interaction strength between the triskelion leg segments was set at $\epsilon=6 k_{B} T$, which lies slightly below the threshold for spontaneous cage formation in the bulk at the chosen clathrin concentration. ${ }^{47}$ Since AP's mid-linker clathrin binding site is not activated in the bulk, cage formation is strictly limited to a small area adjacent to the membrane, as was confirmed by the simulations. The CCP assembly phase diagram in Fig. 10 was obtained by varying both the AP-membrane click strength $\epsilon_{\mathrm{A} m}$ and the AP-clathrin click strength $\epsilon_{\mathrm{CA}}$ over ranges from 4 to $14 k_{B} T$. Each run lasted $1 \mathrm{~s}$, requiring about 10 days of CPU 


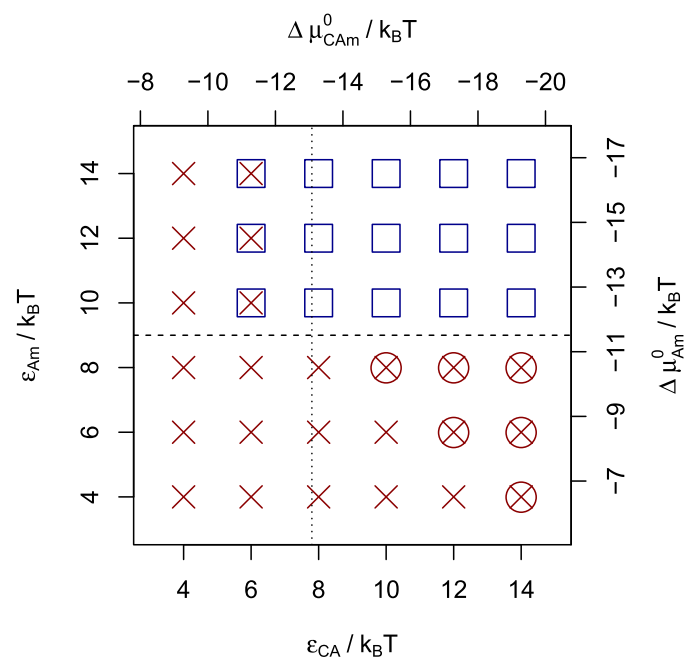

FIG. 10. Phase diagram of clathrin-coat assembly and disassembly at the membrane, as a function of the clathrin-AP clicking strength $\epsilon_{\mathrm{CA}}$ and the membrane-AP clicking strength $\epsilon_{\mathrm{A} m}$. The clathrin and AP bulk concentrations are maintained at $[\mathrm{C}]=10^{-3} \sigma^{-3}$ and $[\mathrm{A}]=10^{-2} \sigma^{-3}$, respectively. Spontaneous assembly within $10^{8}$ time steps (corresponding to $1 \mathrm{~s}$ ) of at least one clathrin pentamer or hexamer ring from solution is marked with empty blue squares, a lack hereof by red crosses. For simulations initiated with a partial coat attached to the membrane, this coat either appears stable (blue squares and blue squares overlaying red crosses), detaches from the membrane and gradually disintegrates (red circles overlaying red crosses) or directly disintegrates (red crosses). Above the dashed line, more than half of the membrane beads are occupied by APs in the absence of clathrin, see Fig. 8. To the right of the dotted line, triskelia in solution are bound to at least one AP on average, by $\beta_{2}$ clicking to the ankles.

time. All simulation boxes were visually inspected for the presence of aggregates with at least one closed ring of triskelia, i.e., a pentamer or hexamer facet of an incipient coat. Every marker in the figure represents results collected from two independent simulations at the same phase point. Clathrin lattices nucleate and grow at the membrane, see the empty blue squares in Fig. 10, when the conditions $\epsilon_{\mathrm{CA}} \gtrsim 7 k_{B} T\left(\Delta \mu_{\mathrm{CA} m}^{0} \lesssim-13 k_{B} T\right)$ and $\epsilon_{\mathrm{A} m} \gtrsim 9 k_{B} T\left(\Delta \mu_{\mathrm{A} m}^{0} \lesssim-12 k_{B} T\right)$ are both met, while no spontaneous assembly was observed outside this region, see the red crosses. Due to the rarity of coat nucleation events at the membrane, mapping a phase diagram by observing spontaneous assembly within a restricted time scale provides an upper limit on the locations of the phase boundaries. A lower limit was obtained by observing the fate of simulations initiated with coated pit structures (grown under conducive conditions); the employed lattice comprised 24 triskelia, forming four hexagonal facets and one pentagonal facet, connected by about 20 APs to a mildly curved membrane. For most parameter combinations, seeded and unseeded systems showed similar final states. The biggest difference between the two sets of simulations occurred for the blue squares inscribed by a red cross in Fig. 10, marking conditions where coats appeared stable and/or growing but their nucleation from solution was too rare to detect. Red circles denote lattices that partly or fully detached from the membrane and subsequently slowly disintegrated. Interestingly, spontaneous coat assembly (empty blue squares) is only observed in simulations where clathrin binds AP2 in solution (to the right of the dotted line), in agreement with experimental data suggesting that clathrin arrives at the membrane with two attached AP2s. ${ }^{30}$
A typical sequence of events during the formation of a coated pit is illustrated in Fig. 11 (Multimedia view). Initially, triskelia diffusing into the neighbourhood of the membrane are tethered to the membrane by APs. Next, triskelia diffusing along the membrane meet and form aggregates. Even small diffusing aggregates have repeatedly been observed to merge into larger aggregates, a process that is rare (and more difficult to spot) in solution. ${ }^{50}$ The first completed ring of triskelia is always a hexagon, probably because the resulting constructan annulus with sprawling unpaired legs-is flatter than a similar construct centered around a pentamer. At this time, the membrane underneath the ring is still essentially flat, save for the perpetual thermal undulations. Small mobile aggregates have a clear preference to reside at membrane sections that match their curvature, as this reduces the elongation of the AP linkers and thereby lowers the free energy. This effect becomes more pronounced when imposing artificial undulations on the membrane shape to mimic the potential effect of, e.g., BAR domains-bending the membrane inward attracts clathrin aggregates - or mutually repulsive cargo moleculesbending the membrane outward repels clathrin aggregates (data not shown). A similar disposition of coats to curved membranes is observed in experiments. ${ }^{94}$ With increasing time, aggregates continue to grow by binding additional APs and triskelia that form additional rings adjacent to previously formed facets. Most lattice patches remain fairly circular; elongated structures arise occasionally, but they often cease to grow and have a tendency to break up into smaller fragments that can grow again. As a patch grows, its extremal segments start to poke into the membrane and consequently the central part of the patch rises. The multiple APs linking the central triskelia to

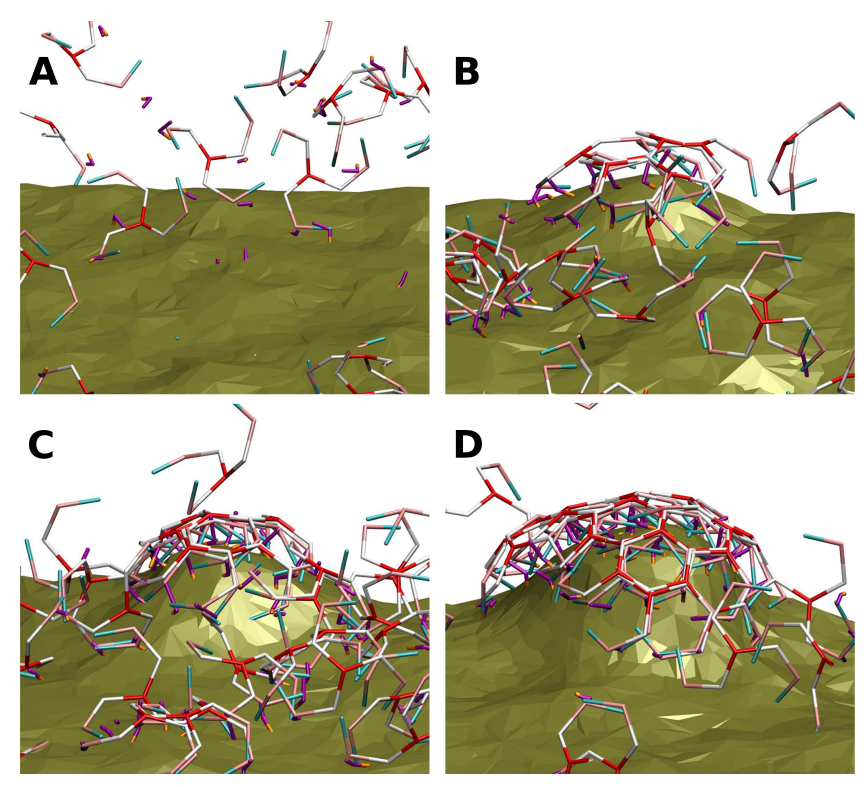

FIG. 11. Snapshots of clathrin triskelia and adaptor proteins spontaneously assembling into a coat at the membrane surface, at time intervals of $0.1 \mathrm{~s}$. The colour scheme is the same as in Fig. 1, with the membrane represented by darkish yellow triangles; the images are taken from a vantage point inside the cell, looking down at the membrane. The interaction strengths are $\epsilon_{\mathrm{CA}}=10 k_{B} T$ and $\epsilon_{\mathrm{A} m}=10 k_{B} T$. A movie of another self-assembly event is available. For presentation purposes, the snapshots and movie were generated with the numbers of triskelia and AP fixed at 60 and 100, respectively, rather than using chemostats, as in the phase diagram of Fig. 10, to stabilize the chemical potentials of these proteins. (Multimedia view) [URL: http://dx.doi.org/10.1063/1.4979985.1] 
the membrane are stretched and eventually lift the membrane below the center of the patch relative to the membrane at the edge of the patch, thus inducing the membrane to smoothly curve toward the lattice and into the cell interior, see Fig. 11(b) (Multimedia view). With the continued growth of the AP-clathrin assembly, a clathrin coated pit is formed, see Figs. 11(c) and 11(d) and the Multimedia view.

The bending resistance by the membrane puts the lattice patch under considerable stress. An additional set of simulations was run to study the stability of the lattice for various bending rigidities exceeding the $10 k_{B} T$ of giant unilamellar vesicles of fluid-phase double-tailed phosphatidylcholine lipids, as expected for biological membranes of more complex lipid compositions and attached proteins. In simulations seeded with the coated pit structures described before, the lattice was stable and/or continued to grow for $k_{\kappa} \lesssim 30 k_{B} T$. At higher values of the bending rigidity, the initially curved membrane largely detached from the lattice and flattened, remaining in contact with the lattice only at its rim; subsequently, these lattices tended to disassemble slowly. On these stiff membranes, the spontaneous assembly of clathrin aggregates ended with the completion of a single hexagonal facet. The unfavourable elongation of the AP tethers connecting the lattice to the unyielding membrane prevented these structures from growing any larger or fusing together. Clearly, this limiting size depends on the pucker angle at the clathrin hub, which we recall to be fixed at $101^{\circ}$ in these simulations, and on the properties of the AP linkers. The stresses exerted by the membrane's bending resistance on the tethered lattice patch are also evidenced by the structure of that lattice. When rigid triskelia with a pucker of $101^{\circ}$ are induced to self-assemble in solution, either by increasing the leg-leg interaction $\epsilon$ or through the help of APs with a permanently active $\beta_{1}$ bead, they form stressminimized near spherical cages of about 60 triskelia with a homogeneous distribution of the pentagonal facets ${ }^{47,51}$ - the assembly appears to be guided by the "exclusion of headto-tail dihedral angle discrepancies"95,96 or "excluded 5566" rule. ${ }^{51}$ The clathrin patches growing against the model membrane, however, typically have developed into a hexagonal facet surrounded by a ring of six hexagonal facets before the first pentagonal facet forms at the edge of the patch. Such a configuration of hexagons is not observed for cages grown in solution with the same $\chi$ but results from the stresses imposed by the tethering to a membrane that resists curvature. Because the initial patch is flattened and denuded in pentagons, it is to be expected that the final cage surrounding a vesicle will be larger than the cages grown in solution with the same $\chi$, the twelve pentagonal facets will be unevenly distributed over the surface of the cage, and hence the cage will be less spherical. Cryo-electron tomography images do indeed show that clathrin cages grown during endocytosis deviate more from a symmetrical shape than empty clathrin cages. ${ }^{6}$

\section{Mechanism}

The role of APs in the aggregation and assembly process is explored by disabling either of their clathrin clicking sites. Since these modified APs can bind one clathrin at most, they cannot gather triskelia in solution and consequently will not induce cage formation in the bulk. But they can still collect

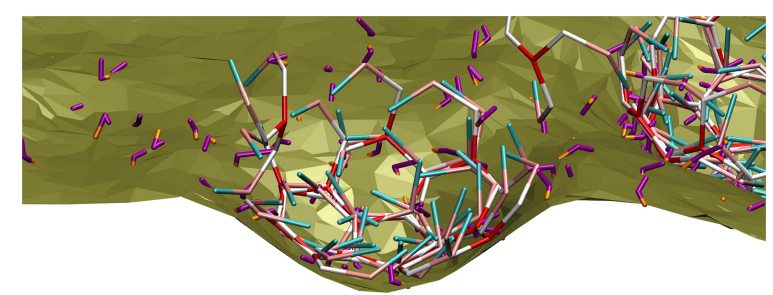

FIG. 12. Clathrin coats formed by mutant APs that bind clathrin only by the appendage $\beta_{2}$ bead clicking to an ankle. The images are again taken from a vantage point inside the cell, looking down at the membrane. While coats formed in the presence of default APs always bend the membrane inward, see Fig. 11 (Multimedia view), the coats compiled by these partially defunctionalized APs systematically cause the membrane to bulge outward.

triskelia at the membrane and thus potentially raise the local clathrin density beyond the critical concentration to spontaneous lattice formation. For APs binding clathrin only through the mid-linker $\beta_{1}$ site clicking to the end of a TD, the coat assembly process still proceeds as described above. Mutant APs binding clathrin only by the appendage $\beta_{2}$ bead clicking to an ankle are also capable of assembling coats adjacent to the membrane, for $\epsilon_{\mathrm{A} m} \gtrsim 10 k_{B} T$ and $\epsilon_{\mathrm{CA}} \gtrsim 10 k_{B} T$. Surprisingly, these coats are systematically oriented upside down: the TDs now point toward the cell interior, rather than to the exterior, and these coats induce evagination of the membrane, rather than the invagination required for endocytosis, as illustrated in Fig. 12. The upside down coats incorporate pentagonal facets at an earlier stage than the functional coats, indicating that the former are under less stress. Upside down patches are also observed in the presence of the default APs when conditions permit lattice assembly to commence in solution. When these fragments land upside-down on a membrane, they typically are anchored by a small number of APs, near the point of closest approach, and consequently hardly bend the membrane on the simulation time scale. Fragments landing the right way up become anchored by APs at the lattice edge but form few membrane connections in the center of the patch and consequently are also inefficient in bending the membrane. We also observed upside-down coats for mutant APs with a functioning $\beta_{1}$ bead, irrespective of the $\beta_{2}$ bead, whose entropic spring constant between $\beta_{0}$ and $\beta_{1}$ has been reduced from $k_{01}=50 k_{B} T / \sigma^{2}$ to $1 k_{B} T / \sigma^{2}$, which approximately triples the
A

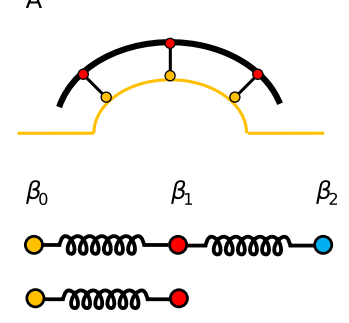

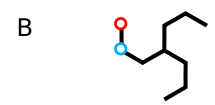
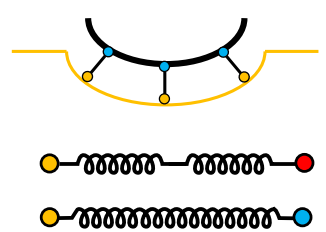

FIG. 13. Cartoons of cross sections (top) of clathrin coats (black line) attached to the cytosolic side of membranes (yellow line) and the adaptor proteins (bottom) giving rise to these coats. Right way up coats (a) are formed when the terminal domain of clathrin (red ring) is bound by an AP $\beta_{1}$ site (red solid circle) that is closely tethered to the AP core (yellow solid circle), irrespective of the AP $\beta_{2}$ site (blue solid circle). Upside down coats (b) are formed by mutant APs whose $\beta_{1}$ site binding the TD is connected to the AP core by a long linker and by mutant APs that only bind with their $\beta_{2}$ site to the clathrin ankle (blue ring). 
linker's average end-to-end length. A visual summary of the AP2-clathrin combinations giving rise to curved membranes is provided in Fig. 13.

\section{DISCUSSION AND CONCLUSIONS}

The nucleation of clathrin coats tethered to a membrane by adaptor proteins has been studied by simulations. This was made possible by combining, adapting, and tuning coarse grained models for the two proteins and the membrane, using both soft interactions and click potentials, simulated by alternating Brownian dynamics and Monte Carlo steps.

Simulations and theory show that the coverage of a membrane by non-aggregating AP2s follows Langmuir-like behaviour. The experimental data by Chang et al. ${ }^{92}$ are also well described by this theory, see Fig. 7 suggesting that aggregation of AP2 plays a minor role. The release of the $\beta_{1}$ clathrin binding site by the AP2 core upon binding of the latter to cargo and membrane can lead to conditions wherein an AP2 core does not bind membrane and cargo in the absence of clathrin but does bind them in the presence of clathrin, see Fig. 8. Along the same line, a small change in a living cell-like the arrival of a cargo molecule-may initiate a sequence of events-like the formation of a clathrin coat-by tipping the thermodynamic stability balance.

The simulations show that the total free energy released by the numerous binding processes during coat formation can be sufficient to bend a membrane into a coated pit. The stress exerted by the membrane on the coat is significant, however. Lattice fragments grown against the membrane are flatter than equally sized fragments in solution, and the formation of pentagonal facets is postponed till a later stage in the growth process. With increasing stiffness of the membrane, the growth of a coat may even cease after a single hexagonal ring has formed because the attachment of additional triskelia results in strong unfavourable steric interactions between these triskelia and the stiff membrane. Of course, a more detailed assessment of whether this process can proceed likewise in vivo requires additional quantitative experimental information on the binding free energies of the proteins involved, on the bending rigidity of clathrin, both as monomers in solution and intertwined in cages, and on the bending strengths and bending resistances of the auxiliary proteins and transmembrane cargo proteins involved.

The rigid clathrin model is based on the triskelion configuration and inter-segmental contacts in cages, and therefore potentially biased to the nucleation-and-growth mechanism of coated pit formation observed in the simulations. Besides experimental data supporting this mechanism, the literature also contains studies reporting coated pit formation by the gradual curling up of pre-existing flat hexagonal clathrin lattices. $^{20,26,33,34,39-42}$ The modifications of the clathrin model required to enable this second mechanism, like the inclusion of flexibility and the reduction of inter segmental binding energies to enable frequent clathrin exchange and lattice reshuffles, are a topic of ongoing research.

The modelled second clathrin motif at the appendix of AP2's $\beta$-linker proves crucial to induce cage assembly in solution, but the simulations indicate that this motif is not required for coat assembly at the membrane. This suggests two alternative assembly mechanisms: (i) in solution the adaptor protein itself brings two triskelia together, while (ii) at the membrane an AP binds to a single triskelion and diffusion along the membrane brings the triskelia together. Since the latter mechanism appears to suffice, it is unclear why AP2s are capable of inducing non-functional cage assembly in solution. We speculate that the $\beta_{2}$ site serves to accelerate the assembly of coats at the membrane.

Comparing the AP models that give rise to normal and upside-down coats, it appears that the competition between steric clathrin-membrane interactions and the elongation of the linker determines the orientation of a coat. For the wild-type short core- $\beta_{1}$ linkers, a triskelion tethered to the membrane by all three legs will experience a significant steric repulsion when oriented the wrong side up, i.e., with its hub sticking into the membrane, and hence there is a strong preference to orient the right way up. Upon binding to neighbouring triskelia, the resulting lattice patch will bend the membrane inward. For mutant APs with longer core- $\beta_{1}$ linkers, and for mutant APs that bind clathrin through $\beta_{2}$ only, this steric repulsion between a triple-tethered upside down triskelion and the membrane is much smaller, and consequently the probability of binding in the wrong orientation is much larger. The simulations suggest that small aggregates formed by these upside down triskelia are under less stress than regular lattices - they show a higher curvature at the same patch size, which indicates they spend less free energy on bending the membrane-and thereby further enhances their stability relative to the functional orientation. To the best of our knowledge, upside-down cages - which do not contribute to endocytosis-have never been observed in in vivo or in vitro experiments. It will be interesting to see whether these upside-down cages can be realised in experiments with mutant AP2s.

\section{ACKNOWLEDGMENTS}

We thank Professor Stefan Luding for stimulating discussions on the membrane model. This work is part of the research programme "self-assembly of protein coats at membranes" (Project No. 711.012.004) which is financed by the Netherlands Organisation for Scientific Research (NWO).

\section{APPENDIX: ADSORPTION AT THE MEMBRANE}

In this appendix, statistical mechanical concepts ${ }^{97}$ are used to derive expressions for the number of APs bound to triskelia and the number of APs and triskelia adsorbed at the membrane. For the reaction of clathrin (C) binding $n$ APs (A),

$$
\mathrm{C}+n \mathrm{~A} \rightleftharpoons \mathrm{CA}_{n},
$$

the equilibrium constant can be defined as

$$
K_{\mathrm{CA}_{n}}=\frac{\left(\left[\mathrm{CA}_{n}\right]_{3 \mathrm{D}} / c_{3 \mathrm{D}}^{0}\right)}{\left([\mathrm{C}]_{3 \mathrm{D}} / c_{3 \mathrm{D}}^{0}\right)\left([\mathrm{A}]_{3 \mathrm{D}} / c_{3 \mathrm{D}}^{0}\right)^{n}}=e^{-\beta \Delta G_{\mathrm{CA}_{n}}^{0}},
$$

where $[\mathrm{X}]_{3 \mathrm{D}}$ denotes the three-dimensional number density of component $\mathrm{X}, c_{3 \mathrm{D}}^{0}$ is a reference concentration typically taken to be $1 \mathrm{M}$, and $\Delta G_{\mathrm{CA}_{n}}^{0}$ is the standard free energy change of the 
reaction. In statistical mechanics, assuming ideal solutions, the above equilibrium constant is calculated as ${ }^{97-99}$

$$
K_{\mathrm{CA}_{n}}=\frac{\left(q_{\mathrm{CA}_{n}} / V\right)}{\left(q_{\mathrm{C}} / V\right)\left(q_{\mathrm{A}} / V\right)^{n}}\left(c_{3 \mathrm{D}}^{0}\right)^{n},
$$

where $q_{\mathrm{X}}$ denotes the molecular partition function of a molecule $\mathrm{X}$ and $V$ the volume of the system. In terms of the chemical potentials of the reaction participants in ideal solutions at the reference concentration,

$$
\mu_{\mathrm{X}}^{0}=-k_{B} T \ln \frac{\left(q_{\mathrm{X}} / V\right)}{c_{3 \mathrm{D}}^{0}},
$$

the standard free energy change of the reaction reads as

$$
\Delta G_{\mathrm{CA}_{n}}^{0}=\mu_{\mathrm{CA}_{n}}^{0}-\mu_{\mathrm{C}}^{0}-n \mu_{\mathrm{A}}^{0}=\Delta \mu_{\mathrm{CA}_{n}}^{0},
$$

with $\Delta \mu_{\mathrm{CA}_{n}}^{0}$ the standard chemical potential change of the reaction.

The partition function of a rigid triskelion in solution follows from the integral of the Boltzmann factor over all particle positions $\mathbf{r}_{\mathrm{C}}$ and orientations $\varphi_{\mathrm{C}}$,

$$
q_{\mathrm{C}}=\frac{1}{\Delta_{\mathrm{C}}} \iint e^{-\beta \Phi} d \mathbf{r}_{\mathrm{C}} d \varphi_{\mathrm{C}} \approx \frac{1}{\Delta_{\mathrm{C}}} 8 \pi^{2} V e^{-\beta \Phi_{\mathrm{C}}},
$$

where $\Delta_{C}$ denotes the elementary volume of the particle (a combination of elementary constants, the temperature, the mass, and the inertia tensor of the triskelion; the exact value is of no consequence as it will drop out in all major results derived below), with $\Phi$ the potential energy, $\Phi_{C}$ the averaged solvation free energy of clathrin and where use was made of the Euler angles with $\varphi_{1} \in[0,2 \pi), \varphi_{2} \in[0, \pi), \varphi_{3} \in[0,2 \pi)$, and $d \varphi_{\mathrm{C}}=\sin \varphi_{2} d \varphi_{1} d \varphi_{2} d \varphi_{3}$.

The AP model described in the main text consists of three beads connected by non-linear springs. The partition function of this molecule reads as

$$
\begin{aligned}
q_{\mathrm{A}} & =\frac{1}{\Delta_{a}^{3}} \iiint e^{-\beta \Phi} d \mathbf{r}_{0} d \mathbf{r}_{1} d \mathbf{r}_{2} \\
& \approx \frac{1}{\Delta_{a}^{3}} e^{-\beta \Phi_{\mathrm{A}}} V q_{s, 01} q_{s, 12},
\end{aligned}
$$

with $\Delta_{a}$ the elementary volume per bead (for simplicity taken to be identical for all beads; again, this factor will cancel out in the final expressions), $\Phi$ the potential energy as a function of the particle positions $\mathbf{r}$, and $\Phi_{\mathrm{A}}$ the average solvation free energy of AP. Integrating over $\mathbf{r}_{2}$ at fixed $\mathbf{r}_{1}$ yields a factor

$$
q_{s, 12}=\int e^{-\beta \psi_{12}\left(r_{12}\right)} d \mathbf{r}_{12} \approx\left(\frac{2 \pi k_{B} T}{k_{12}}\right)^{3 / 2},
$$

where the linker potential $\psi_{12}$ has been approximated by a Hookean spring with spring constant $k_{12}$. One obtains a similar factor when integrating over $\mathbf{r}_{1}$ at fixed $\mathbf{r}_{0}$, and the final integration over $\mathbf{r}_{0}$ yields the volume $V$, collectively arriving at Eq. (A8).

For a dissolved protein complex of a triskelion bound to an AP by the $\beta_{2}$ bead, the partition function reads as

$$
\begin{aligned}
q_{\mathrm{CA}} & =\frac{1}{\Delta_{\mathrm{C}} \Delta_{a}^{3}} \int \cdots \int e^{-\beta \Phi} d \mathbf{r}_{\mathrm{C}} d \varphi_{\mathrm{C}} d \mathbf{r}_{0} d \mathbf{r}_{1} d \mathbf{r}_{2} \\
& \approx \frac{q_{\mathrm{C}} q_{\mathrm{A}}}{V} g_{\frac{4}{3}} \pi \rho_{\mathrm{CA}}^{3} e^{\beta \epsilon_{\mathrm{CA}}},
\end{aligned}
$$

where $\rho_{\mathrm{CA}}$ and $\epsilon_{\mathrm{CA}}$ denote the radius and strength, respectively, of the click interaction between the $\beta_{2}$ bead and any of the $g=3$ matching sites on the triskelion. This remarkably compact result is a consequence of the click potential, which reduces the translational freedom of one of the partners from $V$ to the volume of the click interaction and contributes a Boltzmann factor related to the clicking strength. ${ }^{51}$ This insight is readily extended to triskelia decorated with multiple APs; insertion in Eq. (A3) then yields the equilibrium constants of these clathrin-AP complexes.

If the membrane beads, in their role as binding sites for APs, are both free to diffuse over the entire membrane area $A$ and sufficiently thinly distributed so as to ignore interactions, their partition function will read as

$$
q_{m}=\frac{1}{\Delta_{m}} \int e^{-\beta \Phi} d \mathbf{r}_{m} \approx \frac{1}{\Delta_{m}^{\prime}} A e^{-\beta \Phi_{m}},
$$

with $\Phi_{m}$ the average free energy of a bead, and where the elementary area, $\Delta_{m}^{\prime}$, represents the ratio of the elementary volume for a membrane bead, $\Delta_{m}$, to the mean transverse displacement of a bead confined to a membrane.

The partition function of the complex of an AP molecule clicked by its $\beta_{0}$ bead to a membrane bead is given by

$$
q_{\mathrm{A} m}=\frac{1}{\Delta_{a}^{3} \Delta_{m}} \iiint \int e^{-\beta \Phi} d \mathbf{r}_{0} d \mathbf{r}_{1} d \mathbf{r}_{2} d \mathbf{r}_{m} .
$$

Upon ignoring the non-bonding AP-membrane interactions, i.e., the potential that prevents the $\beta_{1}$ and $\beta_{2}$ beads from crossing the membrane, one readily obtains the approximation, denoted by a tilde,

$$
\tilde{q}_{\mathrm{A} m} \approx q_{m} \frac{q_{\mathrm{A}}}{V} \frac{4}{3} \pi \rho_{\mathrm{A} m}^{3} e^{\beta \epsilon_{\mathrm{A} m}},
$$

where $\rho_{\mathrm{A} m}$ and $\epsilon_{\mathrm{A} m}$ denote the radius and strength, respectively, of the click interaction between the membrane bead and the AP's core. By analogy with the above equilibrium constant in bulk solution, the equilibrium constant of the reaction

$$
\mathrm{A}+m \rightleftharpoons \mathrm{A} m
$$

can be defined as

$$
\begin{aligned}
K_{\mathrm{A} m} & =\frac{\left([\mathrm{A} m]_{2 \mathrm{D}} / c_{2 \mathrm{D}}^{0}\right)}{\left([\mathrm{A}]_{3 \mathrm{D}} / c_{3 \mathrm{D}}^{0}\right)\left([m]_{2 \mathrm{D}} / c_{2 \mathrm{D}}^{0}\right)} \\
& =\frac{\left(q_{\mathrm{A} m} / A\right)}{\left(q_{\mathrm{A}} / V\right)\left(q_{m} / A\right)} c_{3 \mathrm{D}}^{0},
\end{aligned}
$$

where the membrane beads and their complexes are bound to the two-dimensional membrane surface, and $c_{2 \mathrm{D}}^{0}$ denotes a two-dimensional reference concentration. By inserting the preceding expressions for the molecular partition functions, one arrives at

$$
\frac{[\mathrm{A} m]_{2 \mathrm{D}}}{[m]_{2 \mathrm{D}}}=\frac{[\mathrm{A}]_{3 \mathrm{D}}}{c_{3 \mathrm{D}}^{0}} e^{-\beta \Delta G_{\mathrm{Am}}^{0}},
$$

with the free energy change accompanying the reaction given by

$$
\begin{aligned}
\Delta G_{\mathrm{A} m}^{0} & =\mu_{\mathrm{A} m}^{0}-\mu_{m}^{0}-\mu_{\mathrm{A}}^{0}=\Delta \mu_{\mathrm{A} m}^{0} \\
& =-k_{B} T \ln \left(\frac{4}{3} \pi \rho_{\mathrm{A} m}^{3} e^{\beta \epsilon_{\mathrm{A} m}} c_{3 \mathrm{D}}^{0}\right),
\end{aligned}
$$


where the reference chemical potentials of membrane-bound particles and complexes follow from

$$
\mu_{\mathrm{X}}^{0}=-k_{B} T \ln \frac{\left(q_{\mathrm{X}} / A\right)}{c_{2 \mathrm{D}}^{0}} .
$$

Upon noting that $[\mathrm{Am}]_{2 \mathrm{D}}$ and $[\mathrm{m}]_{2 \mathrm{D}}$ are proportional to the fractions of occupied and unoccupied membrane beads, $\theta_{\mathrm{A}}$ and $1-\theta_{\mathrm{A}}$, respectively, one recognizes in Eq. (A18) the Langmuir expression of Eq. (35).

Returning to the partition function of Eq. (A13), the nonbonded interactions of the AP's $\beta_{1}$ and $\beta_{2}$ beads with a locally nearly flat membrane, taken to be the plane $z=0$, can be approximated by the conditions $z_{1} \geq 0$ and $z_{2} \geq 0$. Evaluating the partition function part by part, the integral over $\mathbf{r}_{2}$ at given $\mathbf{r}_{1}$ then yields

$$
\hat{q}_{s, 2}\left(z_{1}\right)=\frac{1}{2}\left(\frac{2 \pi k_{B} T}{k_{12}}\right)^{3 / 2}\left[1+\operatorname{erf}\left(\sqrt{\frac{1}{2} \beta k_{12} z_{1}}\right)\right],
$$

where erf denotes the error function. Assuming $z_{0} \approx 0$ and $k_{01} \approx k_{12}$, the integral over $\mathbf{r}_{1}$ at constant $\mathbf{r}_{0}$ becomes

$$
\hat{q}_{s, 12}=\frac{1}{4}\left(\frac{2 \pi k_{B} T}{k_{12}}\right)^{3}\left(1+\int_{0}^{\infty} \operatorname{erf}(s) e^{-s^{2}} d s\right),
$$

which is readily solved by noting that the Gaussian is the derivative of the error function. The integral over $\mathbf{r}_{0}$ proceeds as before, and by collecting factors we arrive at the remarkably simple result

$$
\hat{q}_{\mathrm{A} m}=\frac{3}{8} \tilde{q}_{\mathrm{A} m},
$$

in excellent agreement with the simulation results in Fig. 6 .

In the AP2 complex and in the simulation model, the midlinker binding site for clathrin, $\beta_{1}$ becomes active when the core, $\beta_{0}$, binds to the membrane. This feature of the model is tested in Section III A, using an analytically solvable simplified model system with only one clathrin binding site per AP, i.e., $\beta_{1}$, and only one matching binding site per triskelion. The partition function of a triskelion bound to an AP which in turn is bound to a membrane bead can be constructed by combining the previous results in this appendix. Partition functions of molecules are typically calculated by integrating the internal and rotational motions relative to the molecule's center of mass, followed by an integration over the position of that reference point.${ }^{97}$ We note that the partition function is independent of the location of the reference point relative to the molecule. In the case under study, it proves advantageous to calculate the partition function of the triskelion with respect to its single AP-binding site. The partition function of an clathrin-AP complex bound to a membrane bead, when ignoring the repulsive non-penetration potential of the membrane, then becomes

$$
\tilde{q}_{\mathrm{CA} m}=\tilde{q}_{\mathrm{A} m} \frac{q_{\mathrm{C}}}{V} \frac{4}{3} \pi \rho_{\mathrm{CA}}^{3} e^{\beta \epsilon_{\mathrm{CA}}} .
$$

The standard free energy change accompanying the reaction

$$
\mathrm{C}+\mathrm{A} m \rightleftharpoons \mathrm{CA} m
$$

then reads as

$$
\begin{aligned}
\Delta G_{\mathrm{CA} m}^{0} & =\mu_{\mathrm{CA} m}^{0}-\mu_{\mathrm{A} m}^{0}-\mu_{\mathrm{C}}^{0}=\Delta \mu_{\mathrm{CA} m}^{0} \\
& =-k_{B} T \ln \left(\frac{4}{3} \pi \rho_{\mathrm{CA}}^{3} e^{\beta \epsilon_{\mathrm{CA}}} c_{3 \mathrm{D}}^{0}\right) .
\end{aligned}
$$

The equilibrium of the two coupled reactions, Eqs. (A15) and (A25), follows by solving the fractional occupancies of membrane beads by APs and AP-clathrin complexes, $\theta_{\mathrm{A}}$ and $\theta_{\mathrm{CA}}$, respectively, from the coupled equations

$$
\begin{gathered}
\frac{[\mathrm{CA} m]_{2 \mathrm{D}}}{[\mathrm{A} m]_{2 \mathrm{D}}}=\frac{\theta_{\mathrm{CA}}}{\theta_{\mathrm{A}}}=\frac{[\mathrm{C}]_{3 \mathrm{D}}}{c_{3 \mathrm{D}}^{0}} e^{-\beta \Delta G_{\mathrm{CA} m}^{0},} \\
\frac{[\mathrm{A} m]_{2 \mathrm{D}}}{[m]_{2 \mathrm{D}}}=\frac{\theta_{\mathrm{A}}}{1-\theta_{\mathrm{A}}-\theta_{\mathrm{CA}}}=\frac{[\mathrm{A}]_{3 \mathrm{D}}}{c_{3 \mathrm{D}}^{0}} e^{-\beta \Delta G_{\mathrm{Am}}^{0},}
\end{gathered}
$$

where in the second line it was used that the fractions of empty and occupied membrane beads add up to unity.

${ }^{1}$ F. M. Brodsky, Annu. Rev. Cell Dev. Biol. 28, 309 (2012).

${ }^{2}$ T. Kirchhausen, D. Owen, and S. C. Harrison, Cold Spring Harbor Perspect. Biol. 6, a016725 (2014)

${ }^{3}$ M. S. Robinson, Traffic 16, 1210 (2015).

${ }^{4}$ E. M. Schmid and H. T. McMahon, Nature 448, 883 (2007).

${ }^{5}$ S. Zaremba and J. H. Keen, J. Cell Biol. 97, 1339 (1983).

${ }^{6}$ Y. Cheng, W. Boll, T. Kirchhausen, S. C. Harrison, and T. Walz, J. Mol. Biol. 365, 892 (2007).

${ }^{7}$ R. A. Crowther and B. M. F. Pearse, J. Cell Biol. 91, 790 (1981).

${ }^{8}$ A. Fotin, Y. Cheng, P. Sliz, N. Grigorieff, S. C. Harrison, T. Kirchhausen, and T. Walz, Nature 432, 573 (2004).

${ }^{9}$ S. K. Lemmon and L. M. Traub, Traffic 13, 511 (2012).

${ }^{10}$ M. P. Czech, Cell 100, 603 (2000).

${ }^{11}$ D. J. Owen, B. M. Collins, and P. R. Evans, Annu. Rev. Cell Dev. Biol. 20, 153 (2004).

${ }^{12}$ L. M. Traub, Nat. Rev. Mol. Cell Biol. 10, 583 (2009).

${ }^{13}$ B. J. Canagarajah, X. Ren, J. S. Bonifacino, and J. H. Hurley, Phys. Scr. 22, 517 (2013).

${ }^{14}$ L. P. Jackson, B. T. Kelly, A. J. McCoy, T. Gaffry, L. C. James, B. M. Collins, S. Höning, P. R. Evans, and D. J. Owen, Cell 141, 1220 (2010).

${ }^{15}$ B. T. Kelly, S. C. Graham, N. Liska, P. N. Dannhauser, S. Höning, E. J. Ungewickell, and D. J. Owen, Science 345, 459 (2014).

${ }^{16}$ C. Knuehl, C. Y. Chen, V. Manalo, P. K. Hwang, N. Ota, and F. M. Brodsky, Traffic 7, 1688 (2006).

${ }^{17}$ H. S. Moskowitz, C. T. Yokoyama, and T. A. Ryan, Mol. Biol. Cell 16, 1769 (2005).

${ }^{18}$ L. Maldonado-Báez and B. Wendland, Trends Cell Biol. 16, 505 (2006).

${ }^{19}$ H. T. McMahon and E. Boucrot, Nat. Rev. Mol. Cell Biol. 12, 517 (2011).

${ }^{20}$ J. Heuser and L. Evans, J. Cell Biol. 84, 560 (1980).

${ }^{21}$ J. E. Heuser, J. H. Keen, L. M. Amende, R. E. Rippoldt, and K. Prased, J. Cell Biol. 105, 1999 (1987).

${ }^{22}$ P. N. Dannhauser, M. Platen, H. Böning, H. Ungewickell, I. A. T. Schaap, and E. J. Ungewickell, Traffic 16, 519 (2015).

${ }^{23}$ I. Gaidarov, F. Santini, R. A. Warren, and J. H. Keen, Nat. Cell Biol. 1, 1 (1999).

${ }^{24}$ X. Wu, X. Zhao, L. Baylor, S. Kaushal, E. Eisenberg, and L. E. Greene, J. Cell Biol. 155, 291 (2001).

${ }^{25}$ D. Loerke, M. Wienisch, O. Kochubey, and J. Klingaud, Traffic 6, 918 (2006).

${ }^{26}$ O. Avinoam, M. Schorb, C. Beese, J. A. G. Briggs, and M. Kaksonen, Science 348, 1369 (2015).

${ }^{27}$ C. J. Merrifield, D. Perrais, and D. Zenisek, Cell 121, 593 (2005).

${ }^{28}$ D. Loerke, M. Mettlen, S. L. Schmid, and G. Danuser, Traffic 12, 815 (2011).

${ }^{29}$ M. J. Taylor, D. Perrais, and C. J. Merrifield, PLoS Biol. 9, e1000604 (2011).

${ }^{30}$ E. Cocucci, F. Aguet, S. Boulant, and T. Kirchhausen, Cell 150, 495 (2012).

${ }^{31}$ D. Loerke, M. Mettlen, D. Yarar, K. Jaqaman, H. Jaqaman, G. Danuser, and S. L. Schmid, PLoS Biol. 7, e1000057 (2009).

${ }^{32}$ S. H. Hong, C. L. Cortesio, and D. G. Drubin, Cell Rep. 12, 2121 (2015).

${ }^{33}$ Y. Li, L. Shang, and G. U. Nienhaus, Nanoscale 8, 7423 (2016).

${ }^{34}$ J. P. Ferguson, N. M. Willy, S. P. Heidotting, S. D. Huber, M. J. Webber, and C. Kural, J. Cell Biol. 214, 347 (2016).

${ }^{35}$ M. G. J. Ford, I. G. Mills, B. J. Peter, Y. Vallis, G. J. K. Praefcke, P. R. Evans, and H. T. McMahon, Nature 419, 361 (2002).

${ }^{36}$ W. M. Henne, H. M. Kent, M. G. J. Ford, B. G. Hegde, O. Daumke, P. J. G. Butler, R. Mittal, R. Langen, P. R. Evans, and H. T. McMahon, Structure 15, 839 (2007).

${ }^{37}$ P. N. Dannhauser and E. J. Ungewickell, Nat. Cell Biol. 14, 634 (2012). 
${ }^{38}$ J. C. Stachowiak, F. M. Brodsky, and E. A. Miller, Nat. Cell Biol. 15, 1019 (2013).

${ }^{39}$ M. Ehrlich, W. Boll, A. van Oijen, R. Hariharan, K. Chandran, M. L. Nibert, and T. Kirchhausen, Cell 118, 591 (2004).

${ }^{40}$ S. Saffarian, E. Cocucci, and T. Kirchhausen, PLoS Biol. 7, e1000191 (2009).

${ }^{41}$ W. Kukulski, M. Schorb, M. Kaksonen, and J. A. G. Briggs, Cell 150, 508 (2012).

${ }^{42}$ J. Grove, D. J. Metcalf, A. E. Knight, S. T. Wavre-Shapton, T. Sun, E. D. Protonotarios, L. D. Griffin, J. Lippincott-Schwartz, and M. Marsh, Mol. Biol. Cell 25, 3581 (2014).

${ }^{43}$ M. Lampe, S. Vassilopoulos, and C. Merrifield, J. Struct. Biol. 196, 48 (2016).

${ }^{44}$ R. Matthews and C. N. Likos, Soft Matter 9, 5794 (2013).

${ }^{45}$ N. Cordella, T. J. Lampo, N. Melosh, and A. J. Spakowitz, Soft Matter 11, 439 (2015)

${ }^{46}$ J. J. VanDersarl, S. Mehraeen, A. P. Schoen, S. C. Heilshorn, A. J. Spakowitz, and N. A. Melosh, Soft Matter 10, 6219 (2014).

${ }^{47}$ W. K. den Otter, M. R. Renes, and W. J. Briels, J. Phys.: Condens. Matter 22, 104103 (2010).

${ }^{48}$ W. K. den Otter, M. R. Renes, and W. J. Briels, Biophys. J. 99, 1231 (2010).

${ }^{49}$ W. K. den Otter and W. J. Briels, Traffic 12, 1407 (2011).

${ }^{50}$ I. M. Ilie, W. K. den Otter, and W. J. Briels, J. Chem. Phys. 141, 065101 (2014).

${ }^{51}$ M. Giani, W. K. den Otter, and W. J. Briels, Biophys. J. 111, 222 (2016).

${ }^{52}$ L. Moshkanbaryans, J. Xue, J. R. Wark, P. J. Robinson, and M. E. Graham, PLoS One 11, e0162050 (2016).

${ }^{53}$ B. Goud, C. Huet, and D. Louvard, J. Cell Biol. 100, 521 (1985).

${ }^{54}$ S. J. Doxsey, F. M. Brodsky, G. S. Blank, and A. Helenius, Cell 50, 453 (1987).

${ }^{55}$ F. J. López-Murcia, S. J. Royle, and A. Llobet, J. Neurosci. 34, 8618 (2014).

${ }^{56}$ K. A. Beck and J. H. Keen, J. Biol. Chem. 266, 4437 (1991).

${ }^{57}$ W. Hao, Z. Luo, L. Zheng, K. Prasad, and E. M. Lafer, J. Biol. Chem. 274, 22785 (1999).

${ }^{58}$ M. Skruzny, A. Desfosses, S. Prinz, S. O. Dodonova, A. Gieras, C. Uetrecht, A. J. Jakobi, M. Abella, W. J. H. Hagen, J. Schulz, R. Meijers, V. Rybin, J. A. G. Briggs, C. Sachse, and M. Kaksonen, Dev. Cell 33, 150 (2015).

${ }^{59}$ J. H. Keen, K. A. Beck, T. Kirchhausen, and T. Jarrett, J. Biol. Chem. 266, 7950 (1991).

${ }^{60}$ M. A. Cousin and P. J. Robinson, Trends Neurosci. 24, 659 (2001).

${ }^{61}$ G. Hollopeter, J. J. Lange, Y. Zhang, T. N. Vu, M. Gu, M. Ailion, E. J. Lambie, B. D. Slaughter, J. R. Unruh, L. Florens, and E. M. Jorgensen, eLife 3, e03648 (2014).

${ }^{62}$ L. Ma, P. K. Umasankar, A. G. Wrobel, A. Lymar, A. J. McCoy, S. S. Holkar, A. Jha, T. Pradhan-Sundd, S. C. Watkins, D. J. Owen, and L. M. Traub, Dev. Cell 37, 428 (2016).

${ }^{63}$ L. von Kleist, W. Stahlschmidt, H. Bulut, K. Gromova, D. Puchkov, M. J. Robertson, K. A. MacGregor, N. Tomilin, A. Pechstein, N. Chau, M. Chircop, J. Sakoff, J. P. von Kries, W. Saenger, H.-G. Krausslich, O. Shupliakov, P. J. Robinson, A. McCluskey, and V. Haucke, Cell 146, 471 (2011)

${ }^{64}$ J. C. Dawson, J. A. Legg, and L. M. Machesky, Trends Cell Biol. 16, 493 (2006).

${ }^{65}$ H. T. McMahon and E. Boucrot, J. Cell Sci. 128, 1065 (2015).
${ }^{66}$ S. E. Miller, S. Mathiasen, N. A. Bright, F. Pierre, B. T. Kelly, N. Kladt, A. Schauss, C. J. Merrifield, D. Stamou, S. Honing, and D. J. Owen, Dev. Cell 33, 163 (2015).

${ }^{67}$ J. C. Stachowiak, E. M. Schmid, C. J. Ryan, H. S. Ann, D. Y. Sasaki, M. B. Sherman, P. L. Geissler, D. A. Fletcher, and C. C. Hayden, Nat. Cell Biol. 14, 944 (2012).

${ }^{68}$ A. Young, Semin. Cell Dev. Biol. 18, 448 (2007).

${ }^{69}$ M. P. Allen and D. J. Tildesley, Computer Simulation of Liquids (Clarendon Press, New York, NY, USA, 1989).

${ }^{70}$ C. W. Gardiner, Stochastic Methods: A Handbook for the Natural and Social Sciences (Springer, Berlin, Germany, 2009).

${ }^{71}$ I. M. Ilie, W. J. Briels, and W. K. den Otter, J. Chem. Phys. 142, 114103 (2015).

${ }^{72}$ J. Garcia de la Torre, G. del Rio Echenique, and A. Ortega, J. Phys. Chem. B 111, 955 (2007).

${ }^{73}$ D. J. Evans and S. Murad, Mol. Phys. 34, 327 (1977).

${ }^{74}$ D. Boal, Mechanics of the Cell (Cambridge University Press, Cambridge, UK, 2002).

${ }^{75}$ K. Kremer and G. S. Grest, J. Chem. Phys. 92, 5057 (1990).

${ }^{76}$ B. M. Collins, A. J. McCoy, H. M. Kent, P. R. Evans, and D. J. Owen, Cell 109, 523 (2002).

${ }^{77}$ D. P. Landau and K. Binder, A Guide to Monte Carlo Simulations in Statistical Physics (Cambridge University Press, Cambridge, UK, 2000).

${ }^{78}$ D. Frenkel and B. Smit, Understanding Molecular Simulation, 2nd ed. (Academic Press, Inc., Orlando, FL, USA, 2001).

${ }^{79}$ D. Nelson, T. Piran, and S. Weinberg, Statistical Mechanics of Membranes and Surfaces, 2nd ed. (World Scientific Publishing, Singapore, 2004).

${ }^{80}$ D. H. Boal and M. Rao, Phys. Rev. A 46, 3037 (1992).

${ }^{81}$ G. Gompper and D. M. Kroll, J. Phys.: Condens. Matter 9, 8795 (1997).

${ }^{82}$ D. Espriu, Phys. Lett. B 194, 271 (1987).

${ }^{83}$ S.-J. Zhao and J. T. Kindt, EPL 69, 839 (2005).

${ }^{84}$ G. Gompper and D. M. Kroll, J. Phys. I (France) 6, 1305 (1996).

${ }^{85}$ S. A. Shkulipa, W. K. den Otter, and W. J. Briels, Phys. Rev. Lett. 96, 178302 (2006).

${ }^{86}$ W. Rawicz, K. C. Olbrich, T. McIntosh, D. Needham, and E. Evans, Biophys. J. 79, 328 (2000).

${ }^{87}$ R. Goetz, G. Gompper, and R. Lipowsky, Phys. Rev. Lett. 82, 221 (1999).

${ }^{88}$ W. K. den Otter, J. Chem. Phys. 123, 214906 (2005).

${ }^{89}$ E. Evans and W. Rawicz, Phys. Rev. Lett. 64, 2094-2097 (1990).

${ }^{90}$ F. Brochard and J. F. Lennon, J. Phys. (France) 36, 1035 (1975).

${ }^{91}$ A. Imparato, J. Chem. Phys. 124, 154714 (2006).

${ }^{92}$ M. P. Chang, W. G. Mallet, K. E. Mostov, and F. M. Brodsky, EMBO J. 12, 2169 (1993).

${ }^{93}$ P. Atkins and J. de Paula, Physical Chemistry, 9th ed. (Oxford University Press, Oxford, UK, 2010).

${ }^{94}$ T. J. Pucadyil and S. S. Holkar, Mol. Biol. Cell 27, 3156 (2016).

${ }^{95}$ S. Schein and M. Sands-Kidner, Biophys. J. 94, 958 (2008).

${ }^{96}$ S. Schein, M. Sands-Kidner, and T. Friedrich, Biophys. J. 94, 938 (2008).

${ }^{97}$ D. A. McQuarrie, Statistical Mechanics (Harper \& Row Publishers, New York, NY, USA, 1976).

${ }^{98}$ P. C. Nelson, Biological Physics, 1st ed. (W. H. Freeman and Company, New York, NY, USA, 2008).

${ }^{99}$ R. Phillips, J. Kondev, and J. Theriot, Physical Biology of the Cell (Garlan Science, New York, NY, USA, 2009). 